Supporting Information

\title{
The Influence of Electrochemical Aging on Bead-Blasted Nickel Electrodes for the Oxygen Evolution Reaction
}

\author{
Audrey K. Taylor, Alexi L. Pauls, Michael T. Y. Paul, and Byron D. Gates * \\ Department of Chemistry \\ Simon Fraser University \\ 8888 University Drive \\ Burnaby, BC V5A 1S6 (Canada) \\ Telephone Number: (778) 782-8066 \\ Fax Number: (778) 782-3765 \\ Email Address: * bgates@sfu.ca
}

This research was supported in part by the Natural Sciences and Engineering Research Council (NSERC) of Canada (Discovery Grant No. 1077758), the Canada Research Chairs Program (B.D. Gates, Grant No. 950-215846), the Engineered Nickel Catalysts for Electrochemical Clean Energy project administered from Queen's University (Grant No. RGPNM 477963-2015) from the NSERC of Canada Discovery Frontiers Program, and CMC Microsystems (MNT Financial Assistance, Grant No. 5030). This work made use of the 4D LABS (www.4dlabs.ca) and the Center for Soft Materials shared facilities supported by the Canada Foundation for Innovation (CFI), British Columbia Knowledge Development Fund (BCKDF), Western Economic Diversification Canada, and Simon Fraser University. 

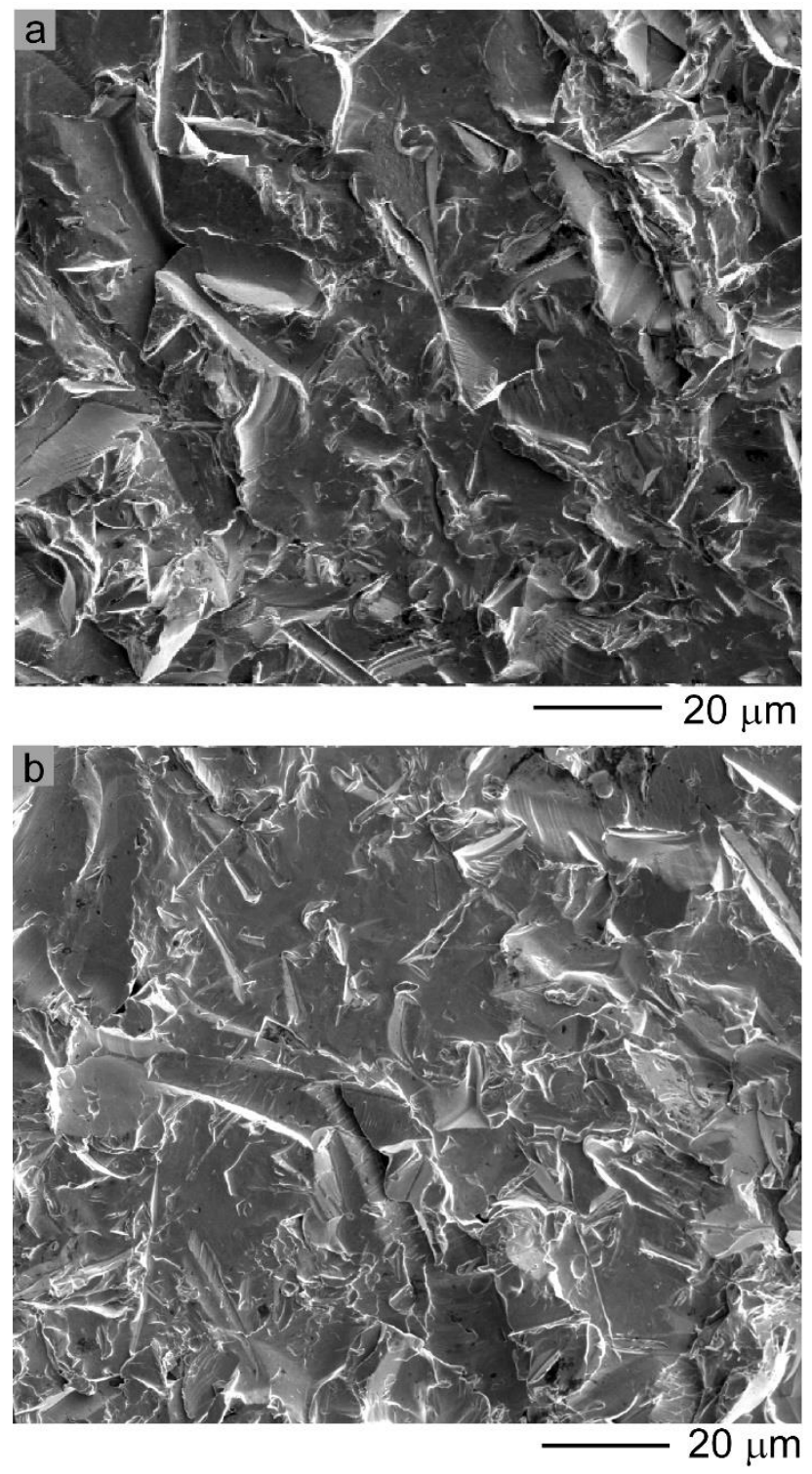

Figure S1. Scanning electron microscopy (SEM) images at high magnification of the (a) electrodeposited Ni electrode after treatment by bead-blasting (BB-edep), and the (b) polished Ni electrode after treatment by the bead-blasting (BB-pol) process. 

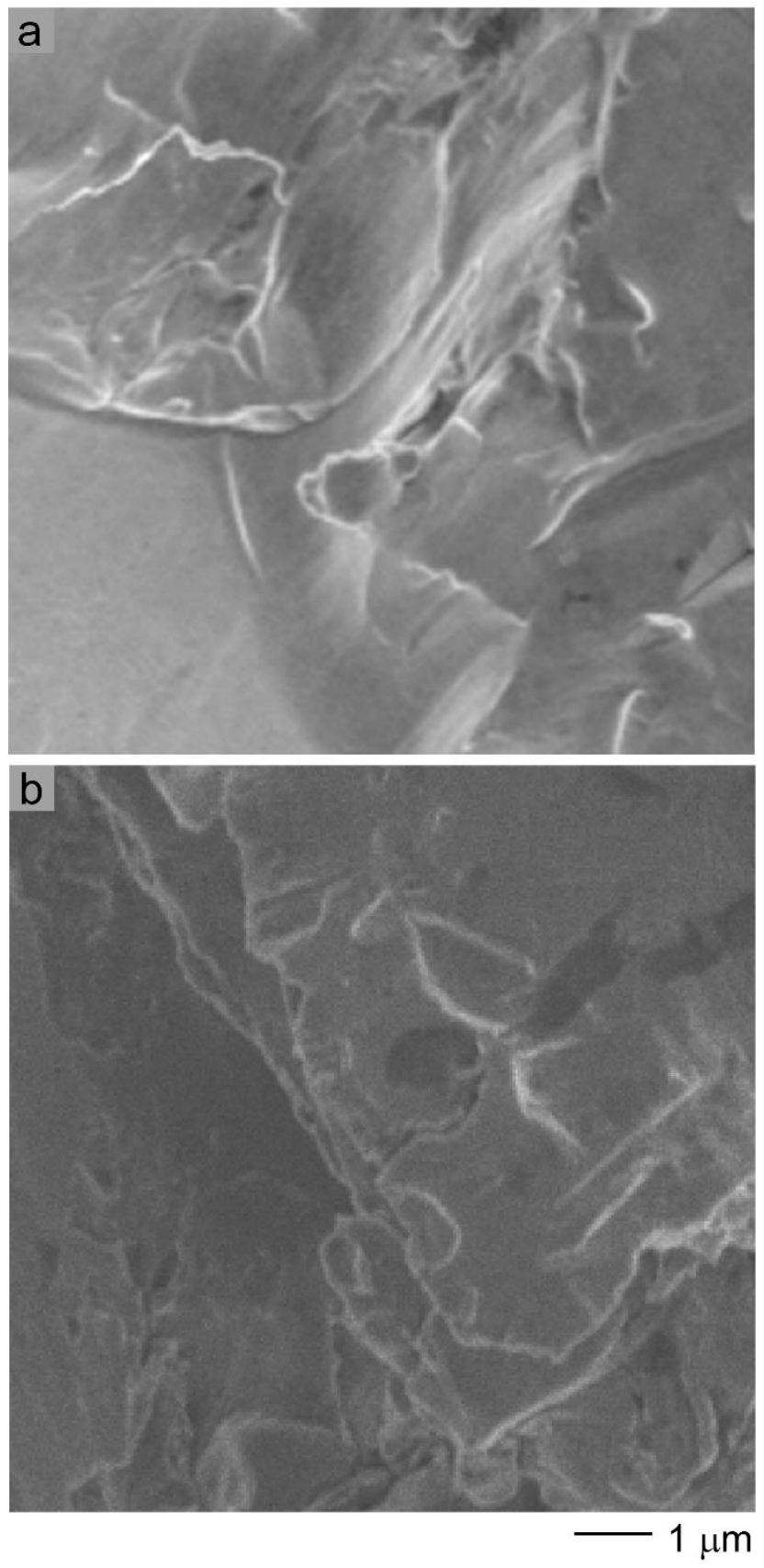

Figure S2. Representative high magnification SEM images of the (a) electrodeposited Ni electrode after treatment by bead-blasting (BB-edep), and the (b) polished Ni electrode after treatment by the bead-blasting (BB-pol) process. 

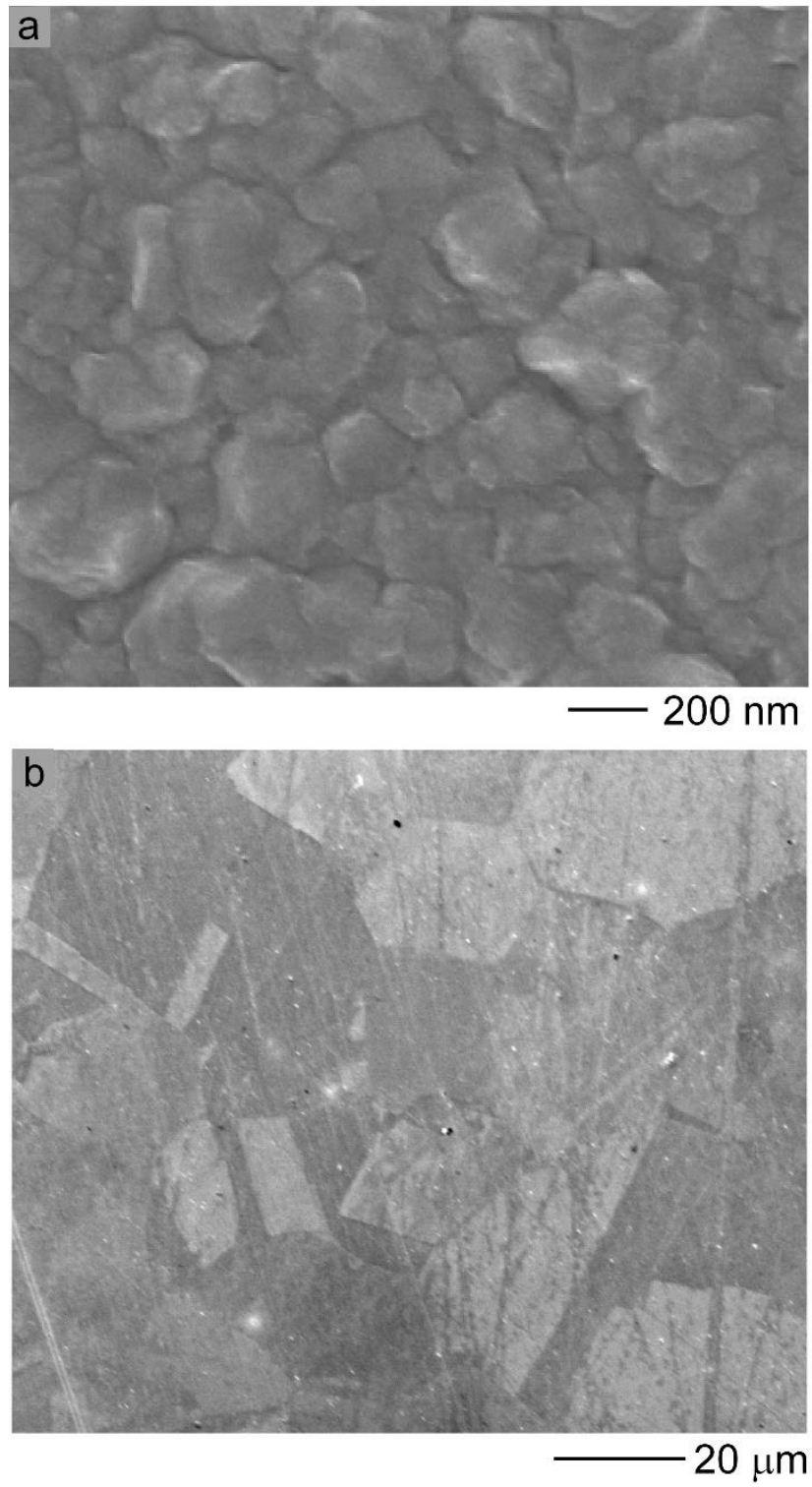

Figure S3. Representative SEM images of (a) the electrodeposited Ni electrode, and (b) the polished Ni electrode at high magnifications. 


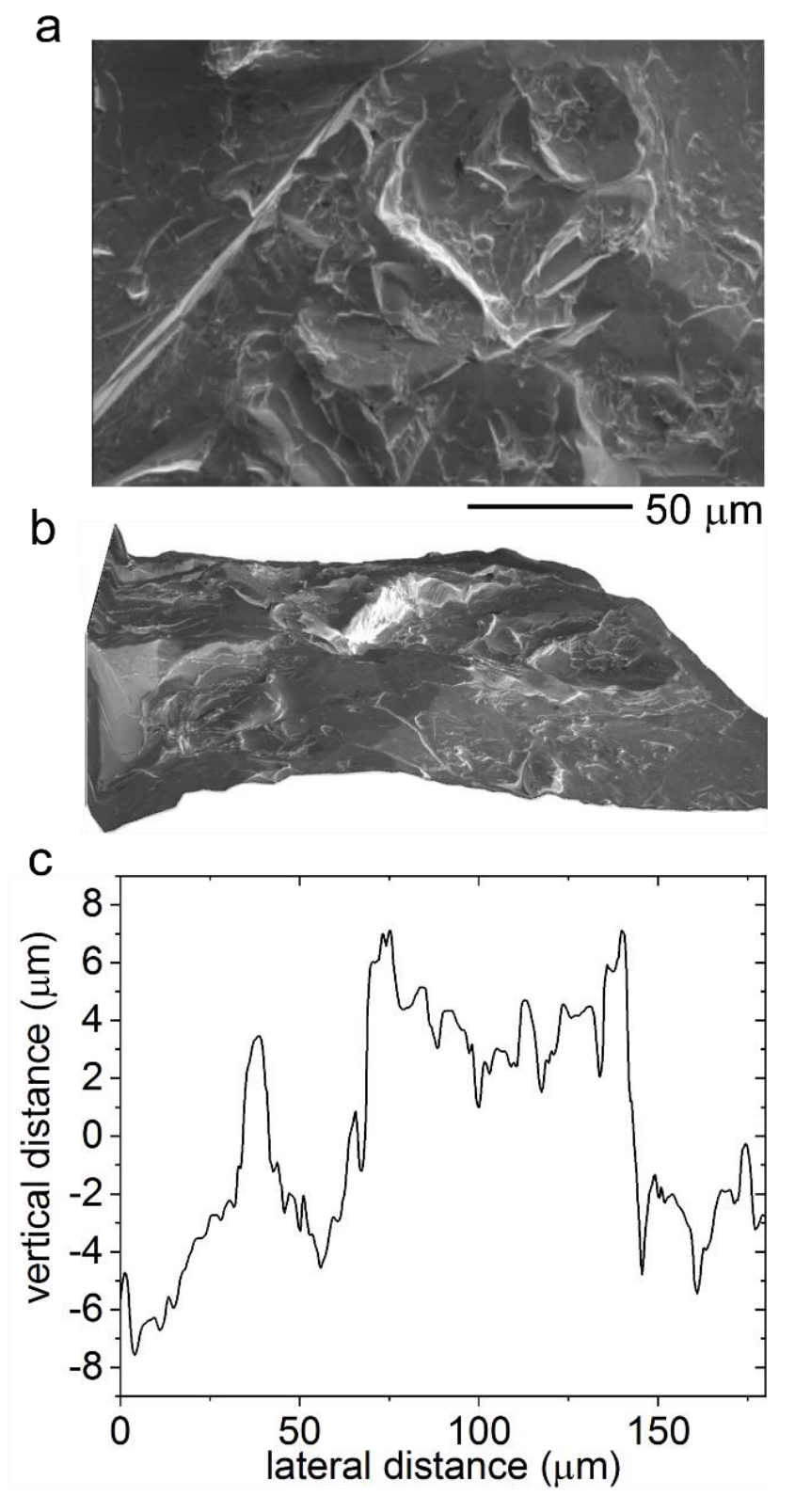

Figure S4. The BB-pol electrode as analysed by SEM to assess the increase in surface area following the bead-blasting process. The image in (a) shows a top-down view of a region analysed using the MountainsMap ${ }^{\circledR}$ software. (b) A three-dimensional (3D) reconstruction of the surfaces from the same region in (a). (c) A representative trace profile resulting from the pixelreconstruction. 

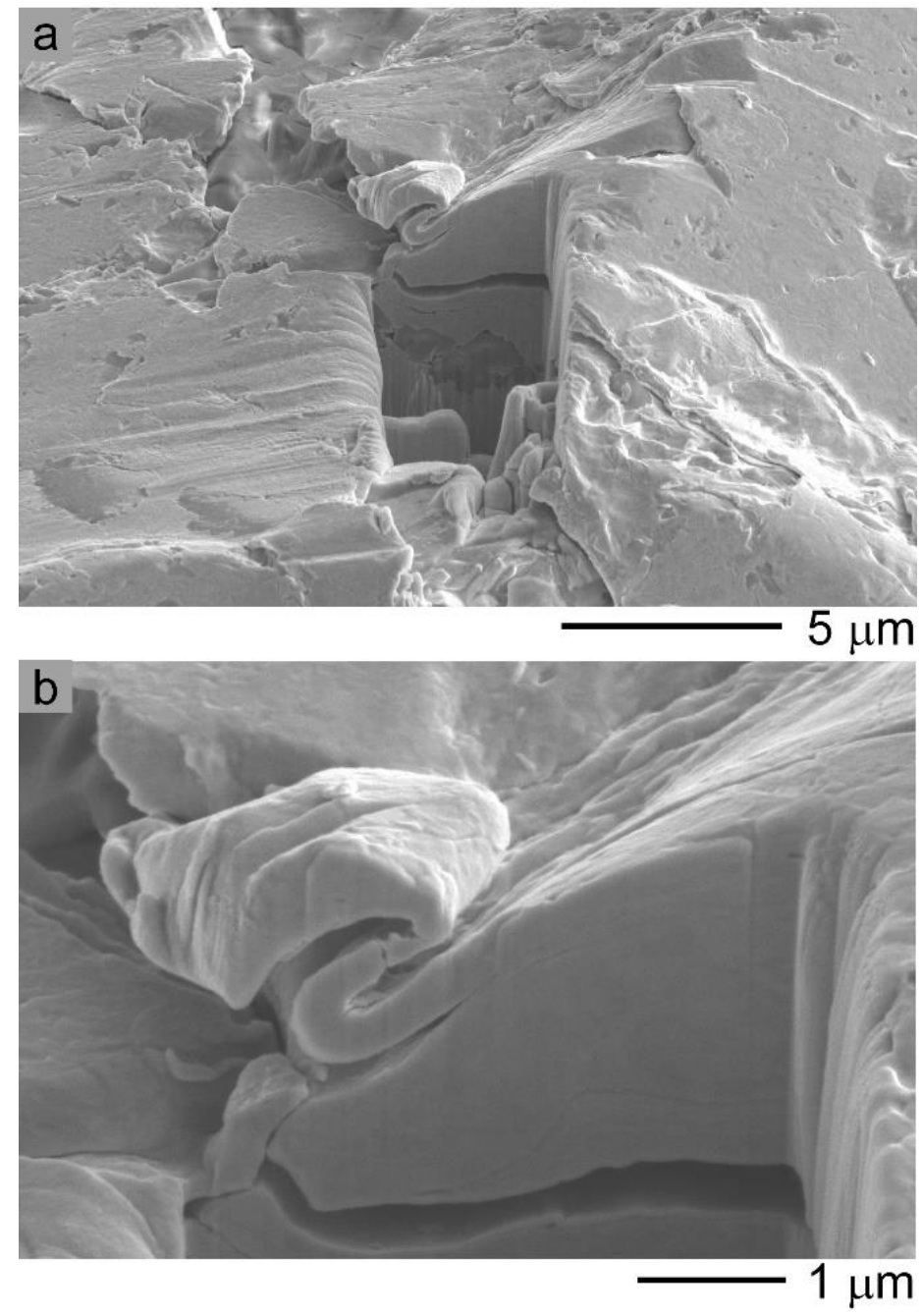

Figure S5. Representative cross-sections prepared by focused ion beam (FIB) milling of the BBedep Ni electrode as imaged by SEM. Secondary electrons were detected at an accelerating voltage of $10 \mathrm{kV}$. The image in (a) shows the region of interest for the milling, and (b) shows this crosssection at a higher magnification. Cracks and buried voids within the electrodeposited layer can be clearly observed in (b). 

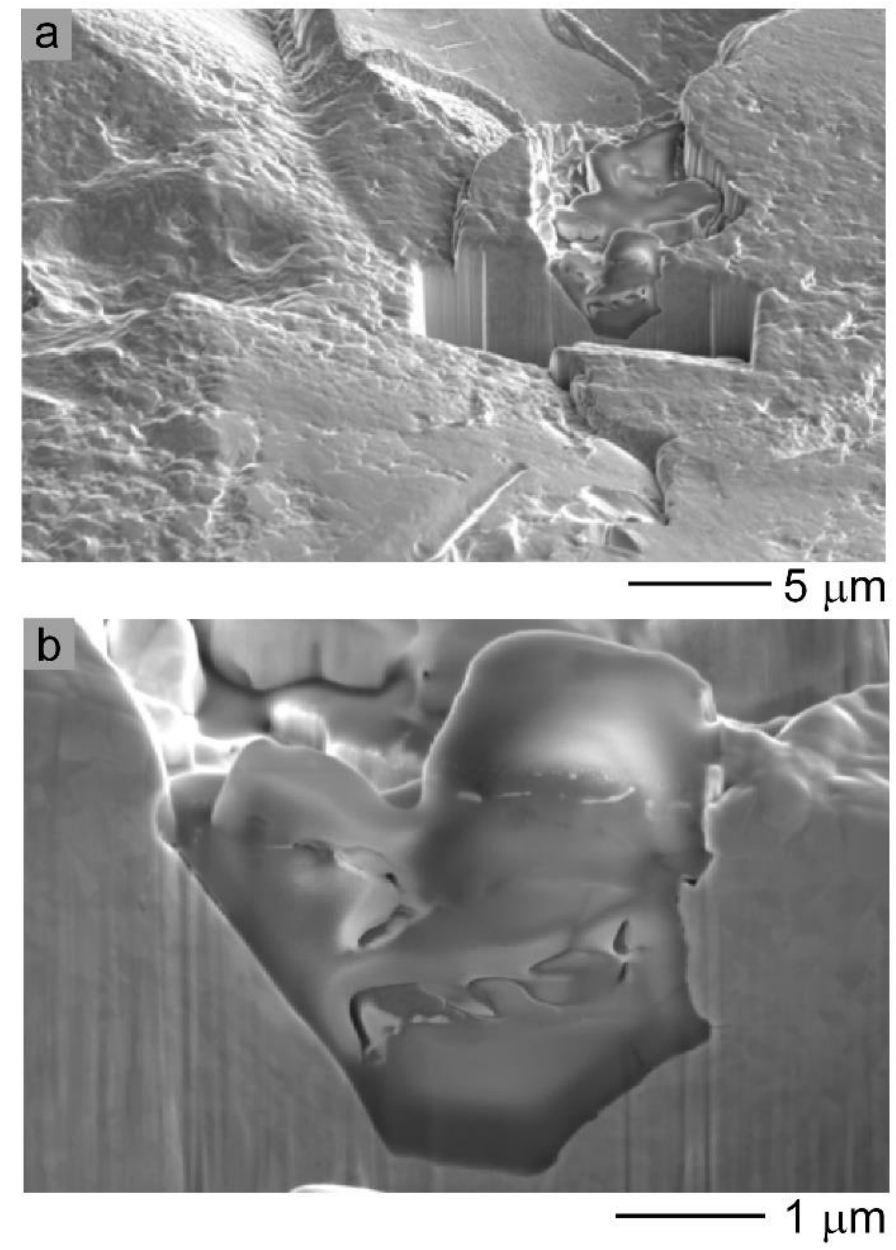

Figure S6. Representative cross-sections prepared by FIB milling of the BB-pol Ni electrode as imaged by SEM. Secondary electrons were detected at an accelerating voltage of $10 \mathrm{kV}$. The image in (a) shows the region of interest for the milling, and (b) shows the cross-section at a higher magnification. Cracks and buried voids can be clearly observed in (b). 

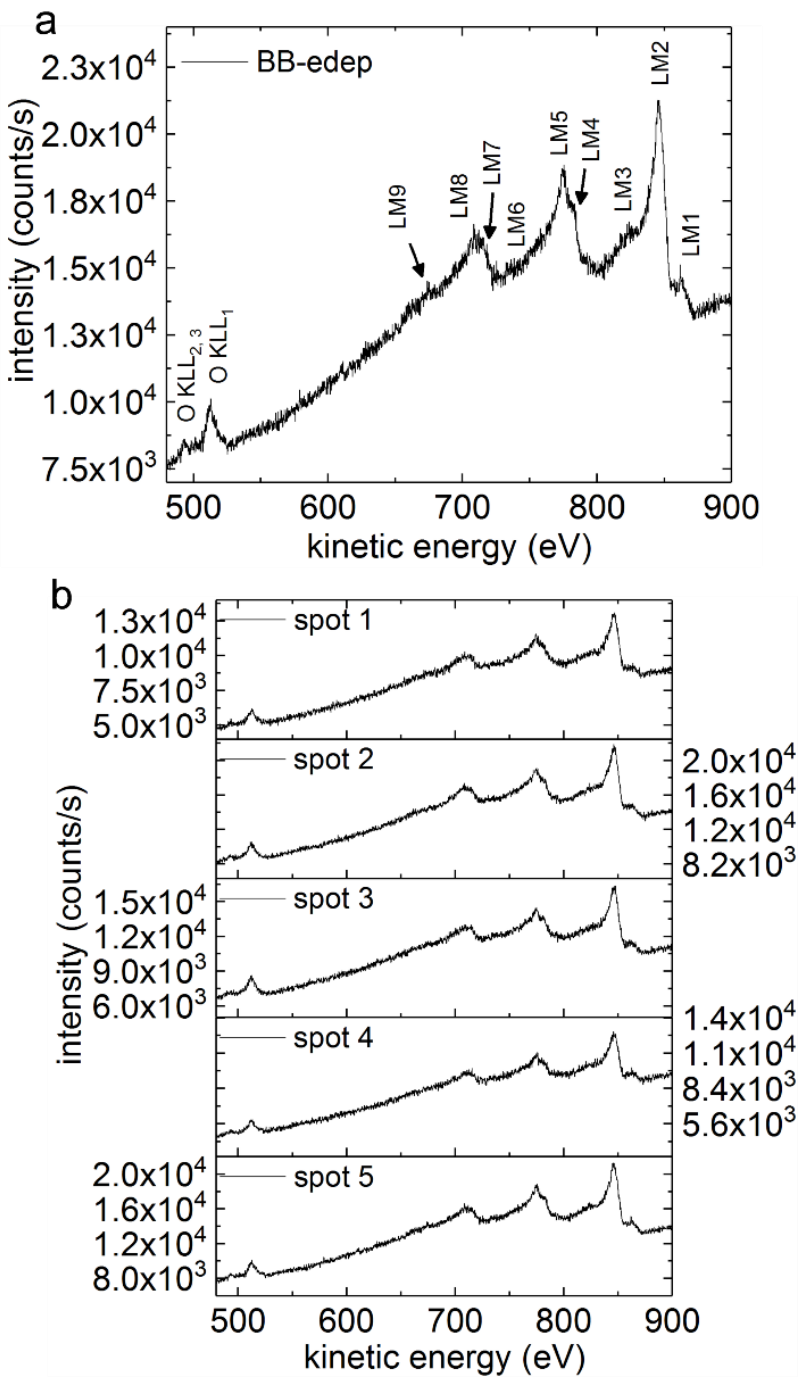

Figure S7. Auger electron spectroscopy (AES) results for the BB-edep electrode after solvent rinsing. The kinetic energy range from 480 to $900 \mathrm{eV}$ contains transitions for many of the first row transition metals. Their energy range was selected to assess the presence of possible surface contamination resulting from the bead-blasting process. The plot in (a) shows a representative AES plot with the labelled transitions for $\mathrm{Ni}$. The data in (b) correspond to the results from five separate regions of the BB-edep electrode. 

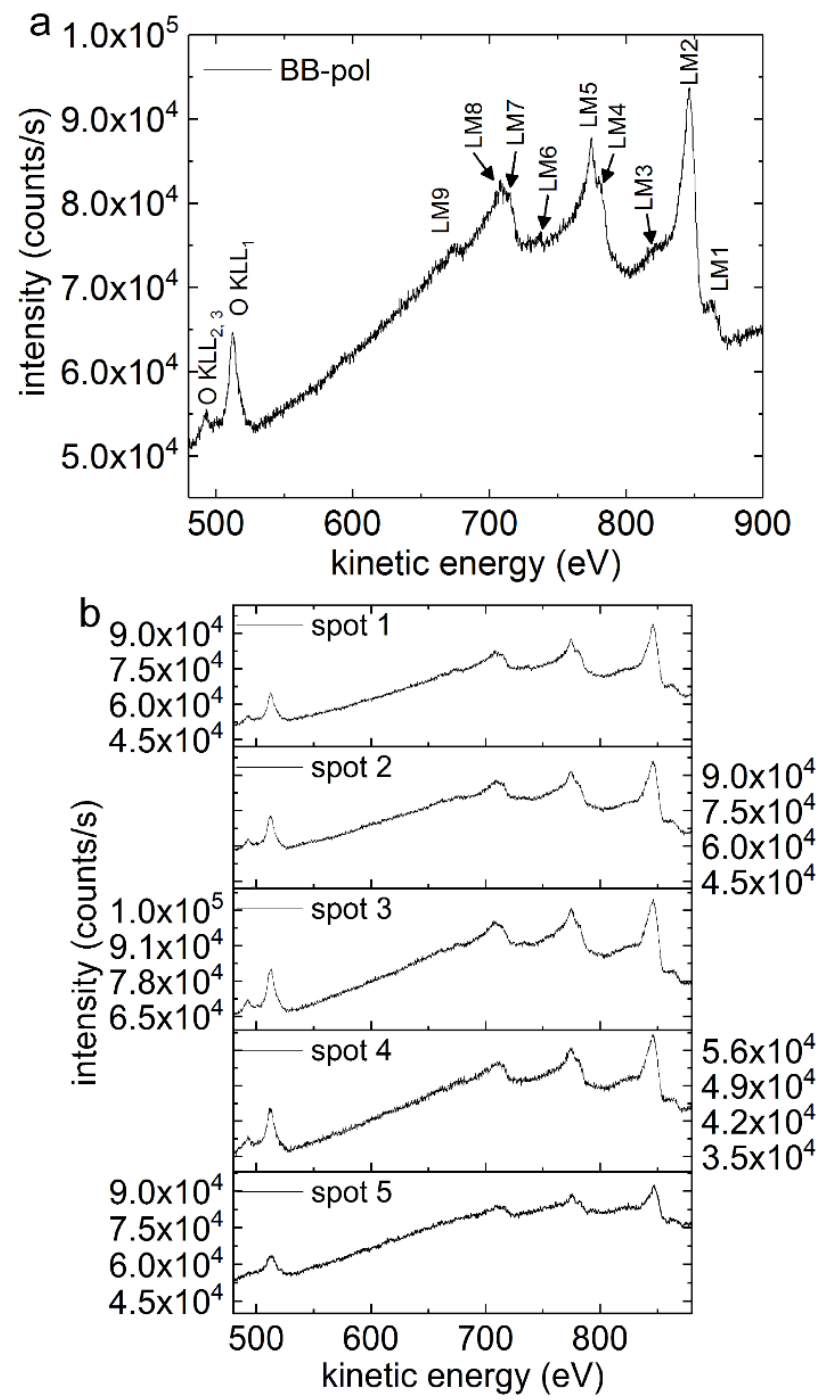

Figure S8. Auger electron spectroscopy results for as-prepared BB-pol electrode after solvent rinsing. The kinetic energy range from 480 to $900 \mathrm{eV}$ was selected for inspection of the presence of possible surface contamination resulting from the bead-blasting process. The plot in (a) shows a representative spectrum with the labelled transitions for Ni. (b) The results from the analysis of five separate regions on the BB-pol electrode. 

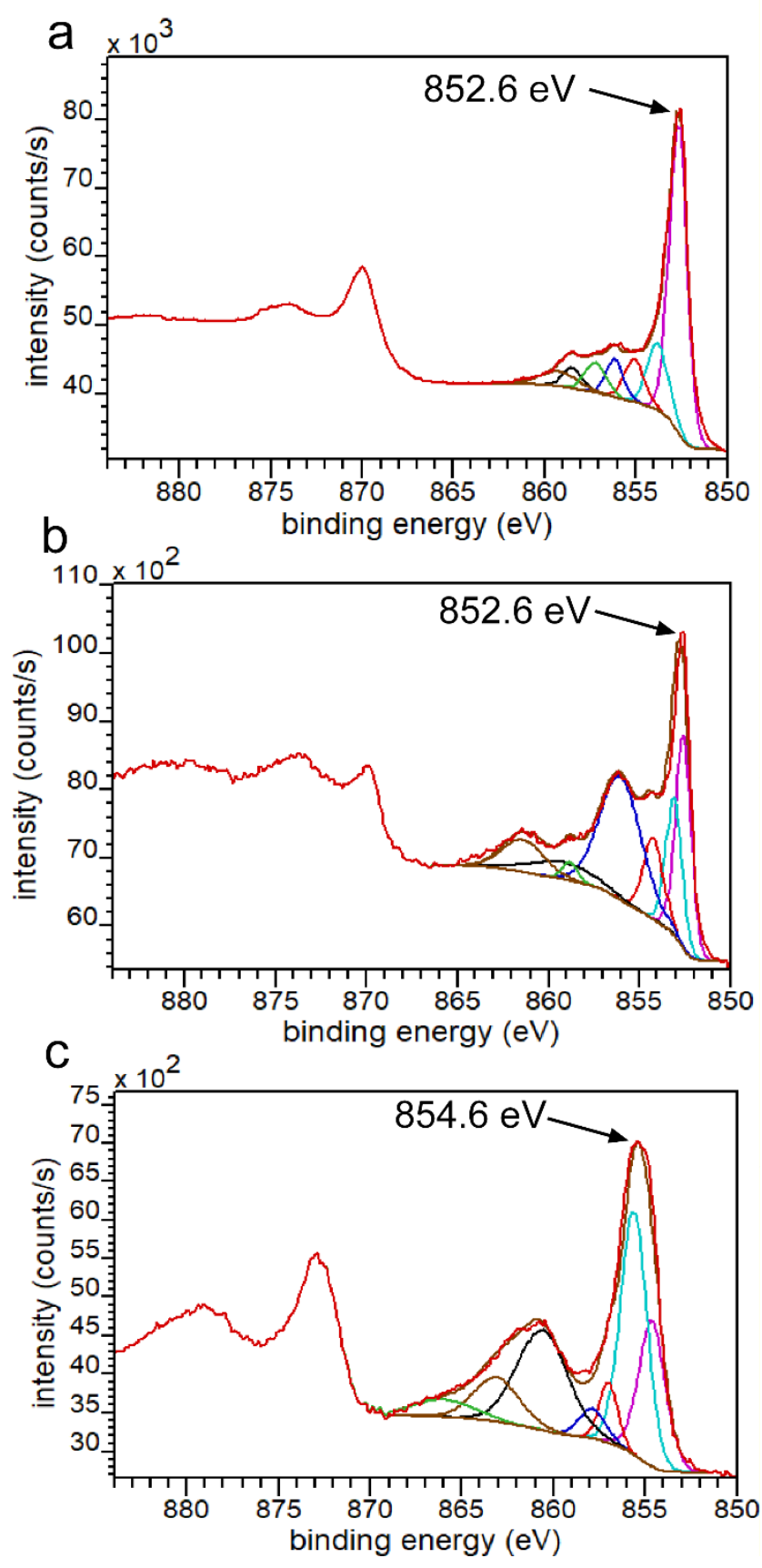

Figure S9. High resolution X-ray photoelectron spectroscopy (HR-XPS) of the Ni 2p region for the (a) electrodeposited $\mathrm{Ni}$, (b) polished $\mathrm{Ni}$, and the (c) BB-pol after the steady-state electrochemical aging treatment. Assignment of the $\mathrm{Ni} 2 \mathrm{p}_{3 / 2}$ region was conducted using the Gupta and Sen multiplet fitting envelopes. ${ }^{1,2}$ 
Table S1. Assignment of chemical species in the HR-XPS for the Ni $2 p_{3 / 2}$ in Figure $S 9$ a-c.

\begin{tabular}{ccc}
\hline chemical component & binding energy $(\mathrm{eV})$ & reference \\
\hline $\mathrm{Ni}$ metal & 852.6 & 1 \\
$\mathrm{NiO}$ & 853.7 & 1 \\
$\mathrm{Ni}(\mathrm{OH})_{2}$ & 854.6 & 2 \\
\hline
\end{tabular}



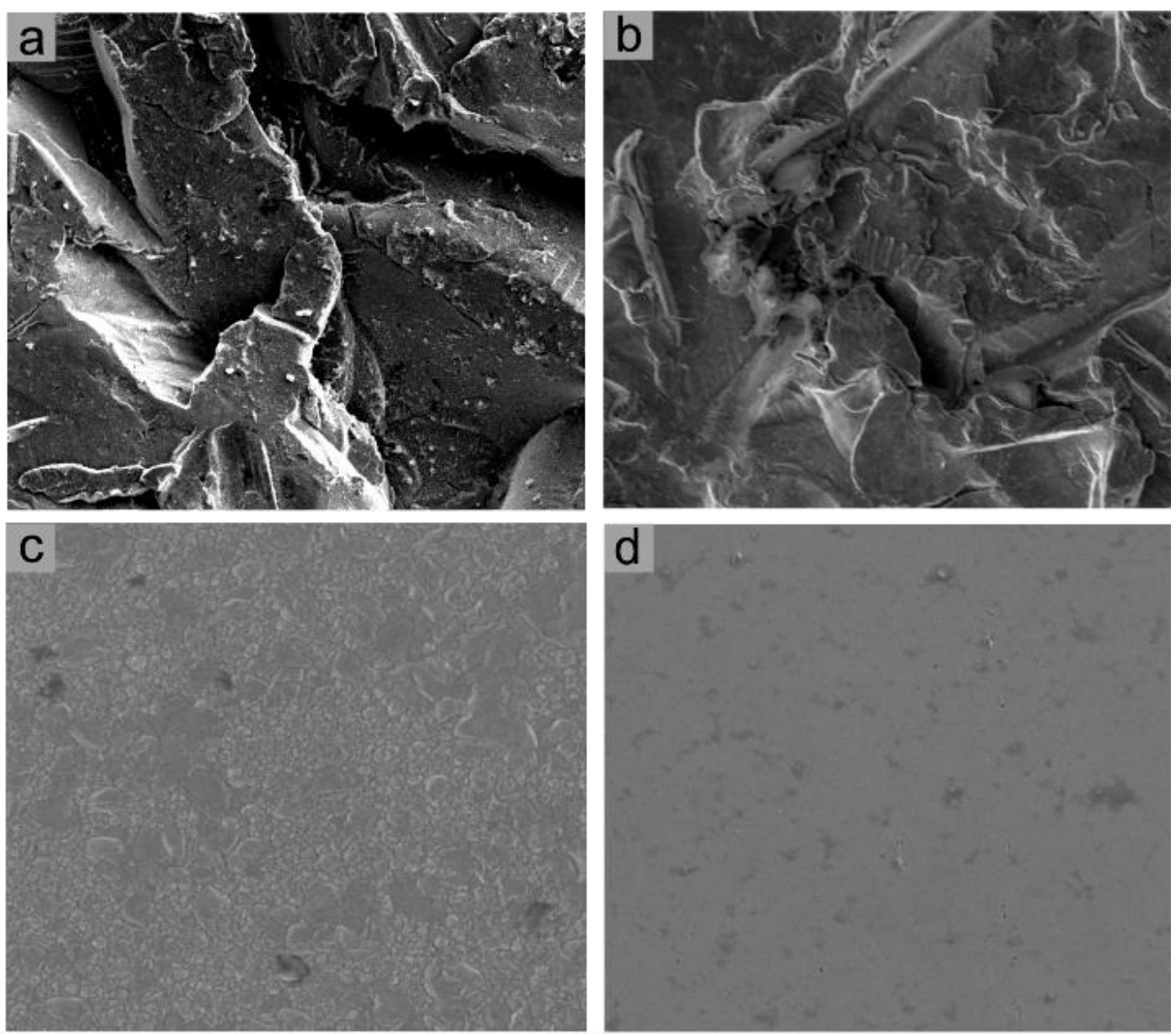

$10 \mu \mathrm{m}$

Figure S10. Representative SEM images of the samples after the steady-state electrochemical aging treatment: (a) BB-edep; (b) BB-pol; (c) planar electrodeposited Ni; and (d) planar polished $\mathrm{Ni}$. 

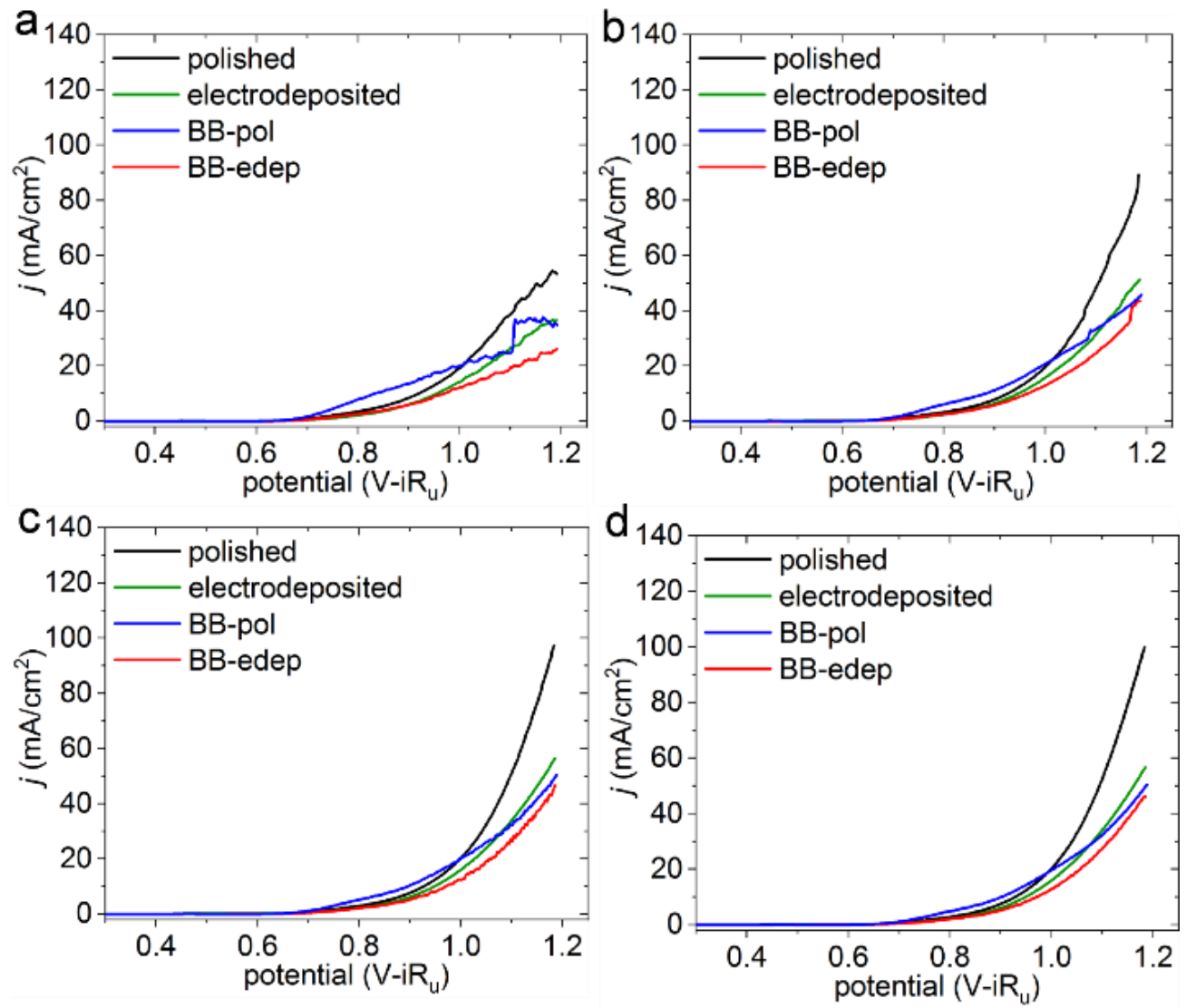

Figure S11. Representative linear sweep voltammetry (LSV) plots for the polished, electrodeposited, BB-pol, and BB-edep Ni electrodes after completing the limited aging process. These LSVs were obtained at several rotational speeds: (a) 0 rpm; (b) $500 \mathrm{rpm}$; (c) $2000 \mathrm{rpm}$; and (d) $3000 \mathrm{rpm}$. The LSVs were recorded at a scan rate of $1 \mathrm{mV} / \mathrm{s}$ in high purity $1 \mathrm{M} \mathrm{KOH}$ at $25^{\circ} \mathrm{C}$. The currents were normalized to the theoretical $\mathrm{SA}_{\mathrm{f}}$. 

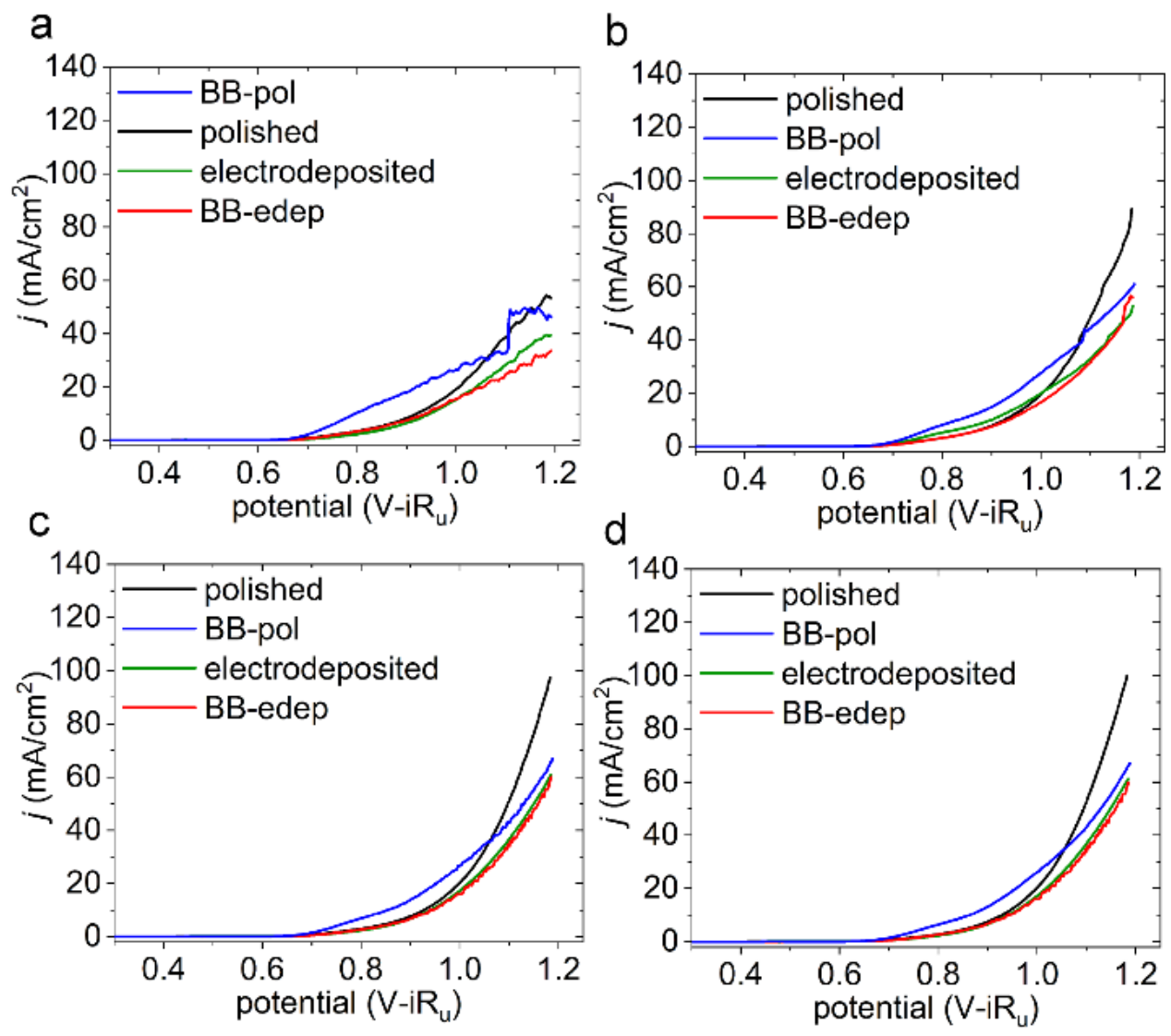

Figure S12. Representative LSV plots for the polished, electrodeposited, BB-pol, and BB-edep Ni electrodes after completing the limited aging process. These LSVs were obtained at several rotational speeds: (a) $0 \mathrm{rpm}$; (b) $500 \mathrm{rpm}$; (c) $2000 \mathrm{rpm}$; and (d) $3000 \mathrm{rpm}$. The LSVs were recorded at a scan rate of $1 \mathrm{mV} / \mathrm{s}$ in high purity $1 \mathrm{M} \mathrm{KOH}$ at $25^{\circ} \mathrm{C}$. The currents were normalized to the geometric area of the electrodes. 

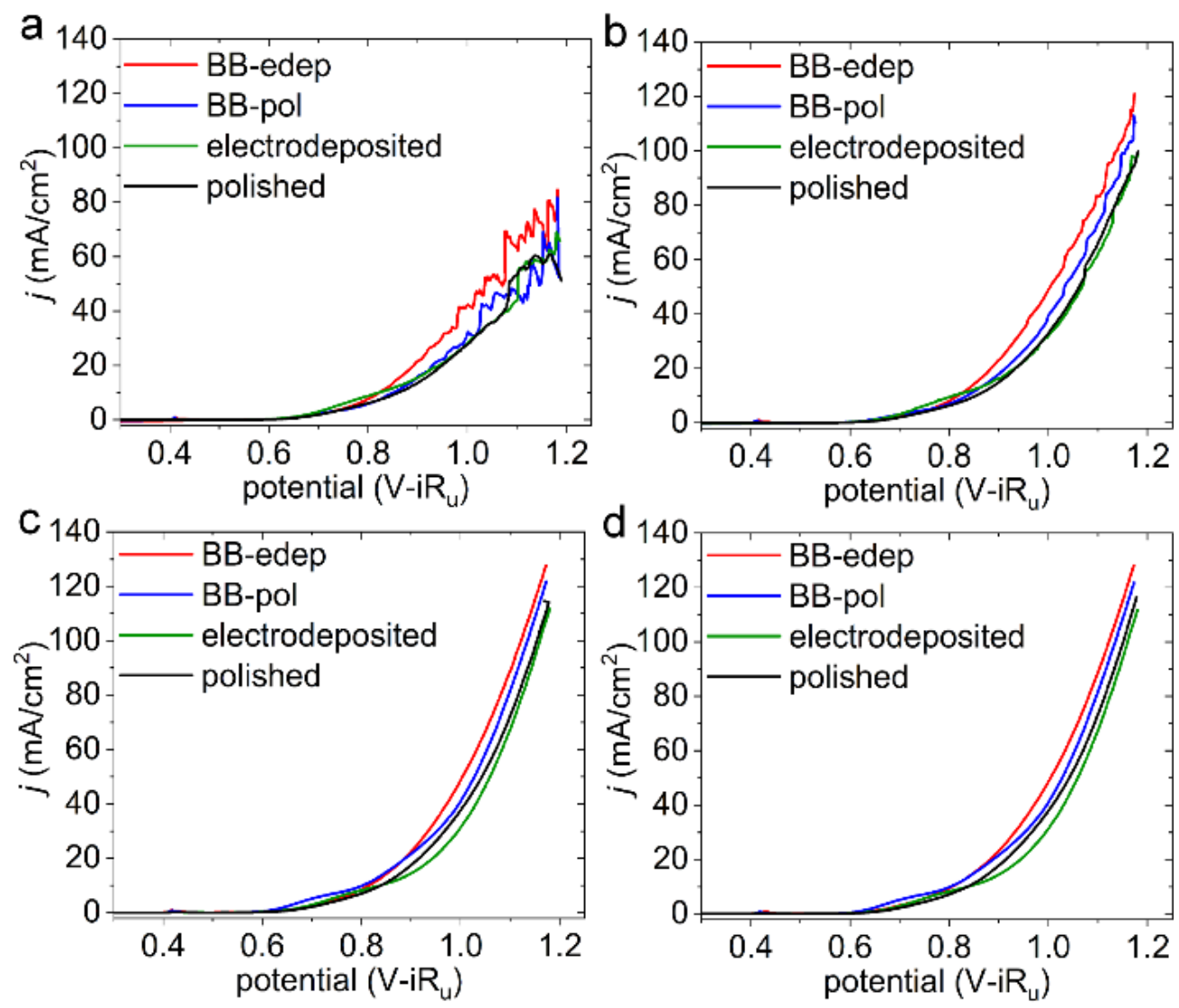

Figure S13. Representative LSV plots for each of the Ni electrodes obtained at different rotational speeds: (a) $0 \mathrm{rpm}$; (b) $500 \mathrm{rpm}$; (c) $2000 \mathrm{rpm}$; and (d) $3000 \mathrm{rpm}$. The LSVs were recorded at a scan rate of $1 \mathrm{mV} / \mathrm{s}$ in high purity $1 \mathrm{M} \mathrm{KOH}$ at $25{ }^{\circ} \mathrm{C}$ after applying the steady-state aging process to each type of electrode. All data were normalized to the theoretical $\mathrm{SA}_{\mathrm{f}}$. 
a
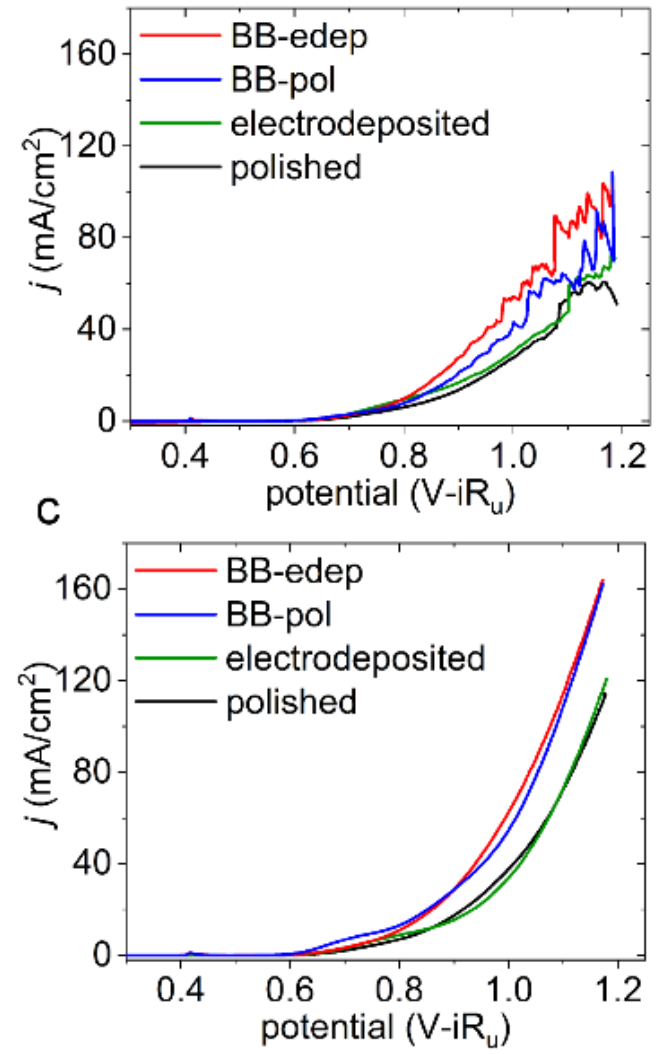

b
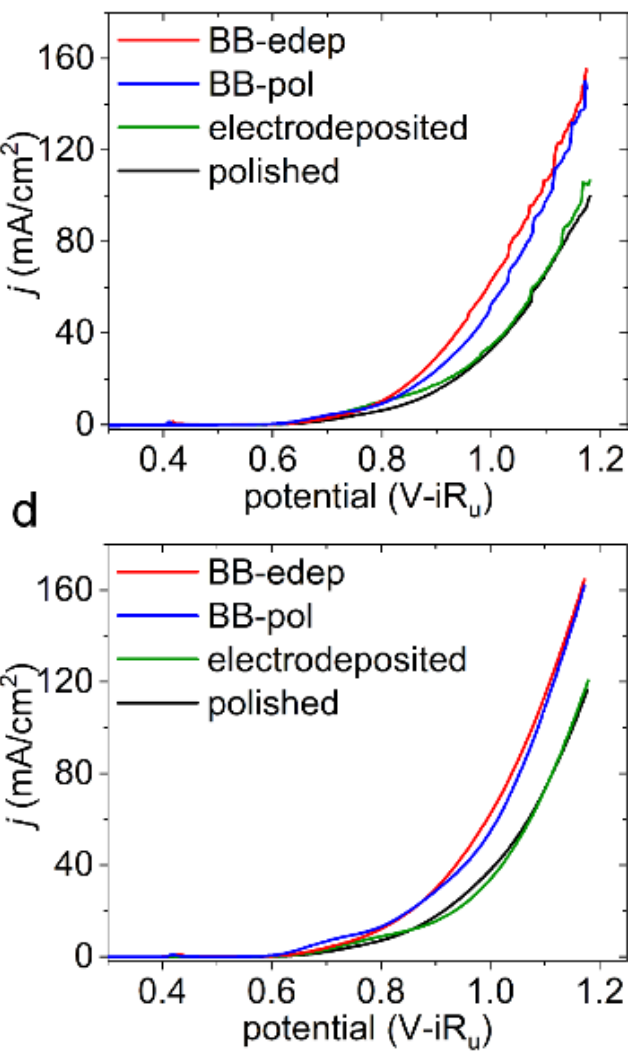

Figure S14. Representative LSV plots for each of the Ni electrodes obtained at different rotational speeds: (a) $0 \mathrm{rpm}$; (b) $500 \mathrm{rpm}$; (c) $2000 \mathrm{rpm}$; and (d) $3000 \mathrm{rpm}$. The LSVs were recorded at a scan rate of $1 \mathrm{mV} / \mathrm{s}$ in high purity $1 \mathrm{M} \mathrm{KOH}$ at $25{ }^{\circ} \mathrm{C}$ after applying the steady-state aging process to each type of electrode. The currents were normalized to the geometric area of the electrodes. 

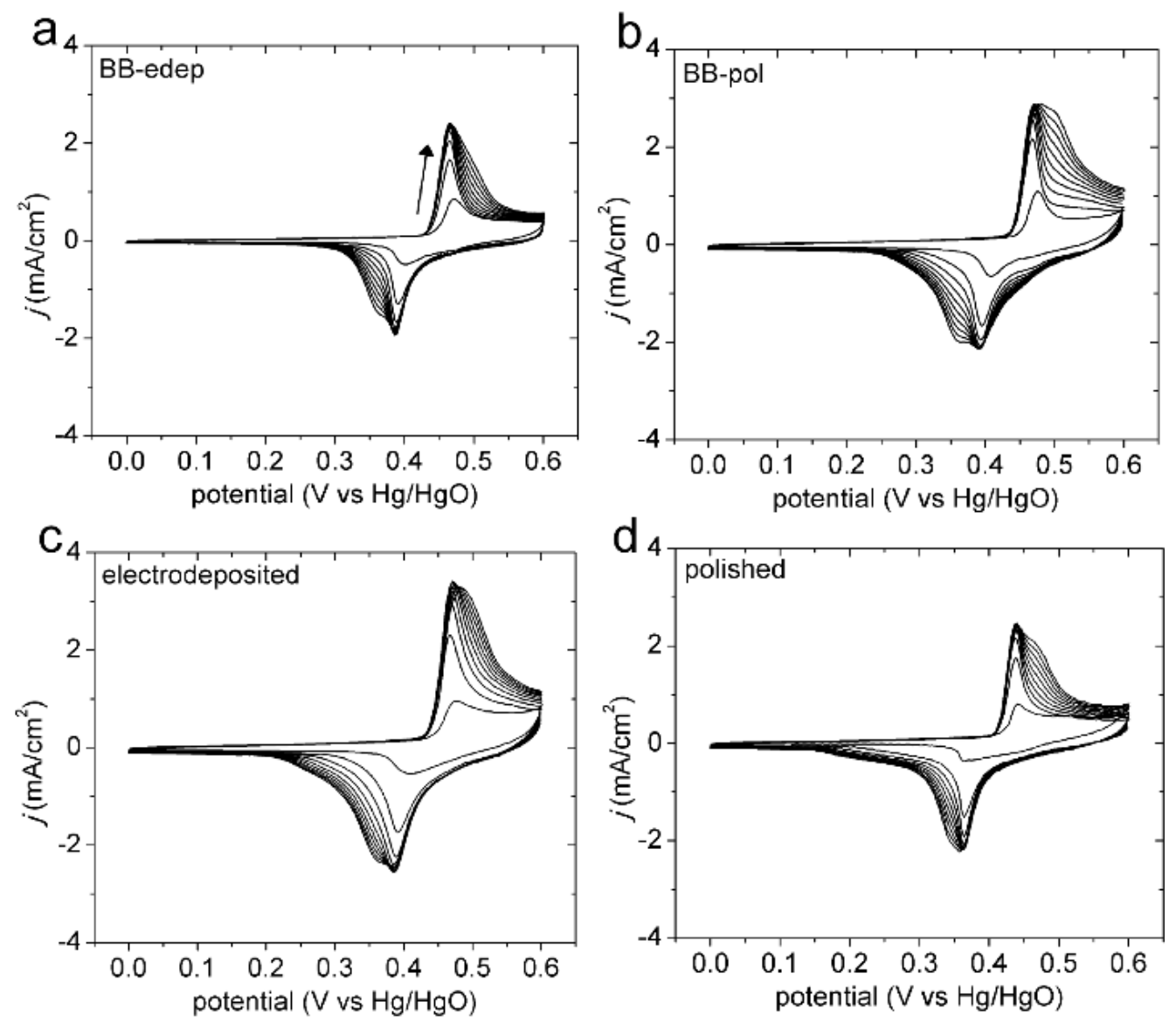

Figure S15. Representative cyclic voltammetry (CV) measurements obtained during electrochemical aging of the $\mathrm{Ni}$ electrodes showing every $10^{\text {th }}$ scan, which are normalized to the geometric areas of the electrodes. Data are plotted for: (a) BB electrodeposited $\mathrm{Ni}$; (b) BB polished $\mathrm{Ni}$; (c) electrodeposited $\mathrm{Ni}$; and (d) polished $\mathrm{Ni}$. The CVs were acquired while immersing the electrodes in high purity $1 \mathrm{M} \mathrm{KOH}$ and using a scan rate of $100 \mathrm{mV} / \mathrm{s}$ at $25^{\circ} \mathrm{C}$. The arrow shown in (a) indicates the direction of the change to the anodic peak with progressive scans. These working electrodes were each rotated at $5500 \mathrm{rpm}$ throughout the CV experiments. A total of 100 CV scans were acquired as per the limited aging process. 

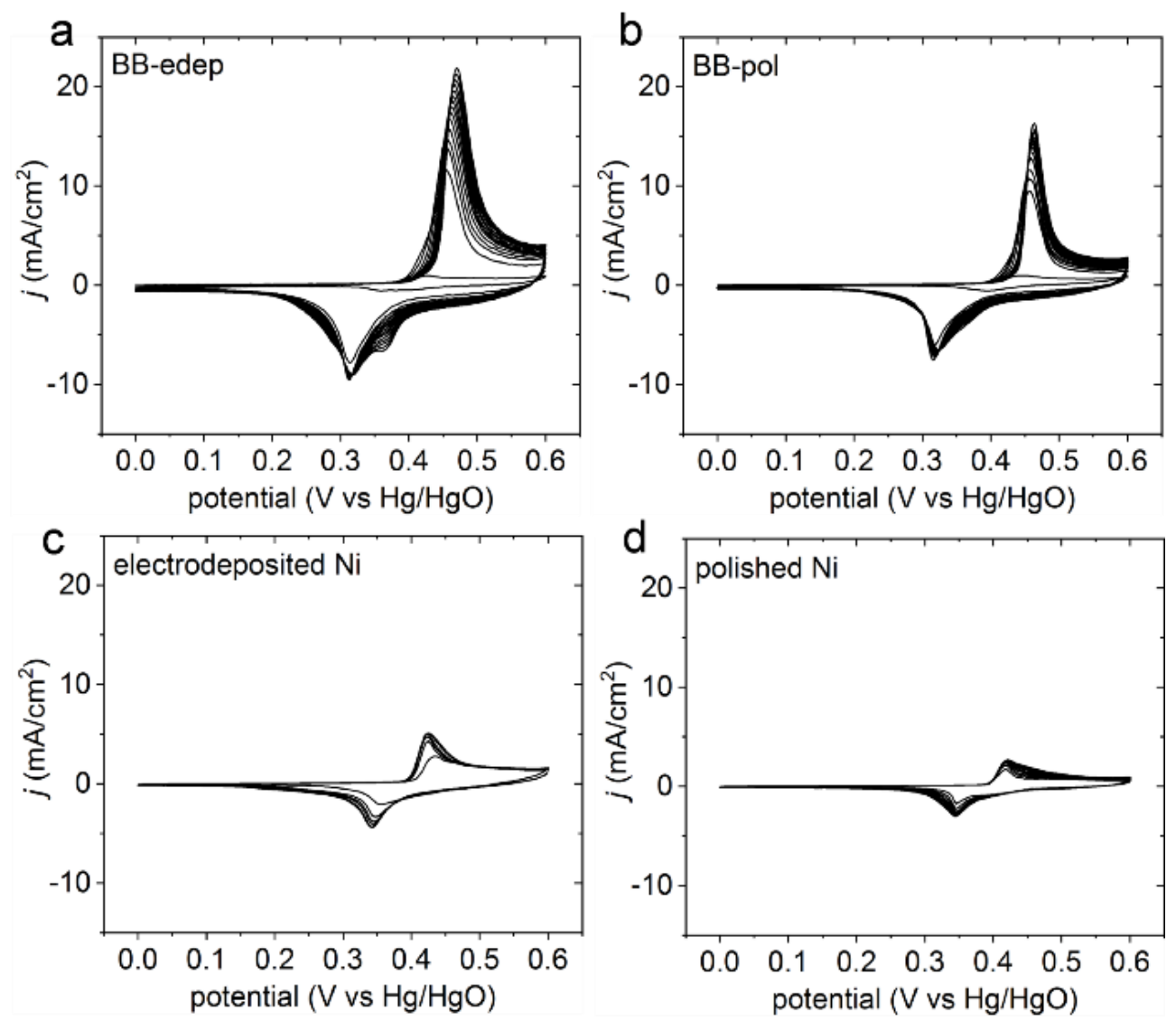

Figure S16. Electrochemical aging by $\mathrm{CV}$ techniques using the steady-state aging process. The steady-state aging sequence required $7200 \mathrm{CV}$ scans for the BB-edep and BB-pol electrodes, while less than $100 \mathrm{CV}$ scans were required for the planar electrodeposited and polished $\mathrm{Ni}$ electrodes. Every $500^{\text {th }} \mathrm{CV}$ scan is shown for the (a) BB-edep and (b) the BB-pol samples. Every $10^{\text {th }} \mathrm{CV}$ scan is shown for the (c) electrodeposited and (d) polished Ni samples. The scan rate was held at $100 \mathrm{mV} / \mathrm{s}$ while the working Ni electrodes were immersed in purified $1 \mathrm{M} \mathrm{KOH}$ at $25^{\circ} \mathrm{C}$. The data were normalized to the geometric surface area of each electrodes. 
Table S2. Charge values obtained from the cathodic reduction peaks in the CV profiles.

\begin{tabular}{ccc}
\hline electrode type* & steady-state aging, $Q_{\text {cat }}(\mathrm{mC})^{\dagger}$ & limited aging, avg $Q_{\text {cat }}(\mathrm{mC})^{\ddagger}$ \\
\hline BB-edep & 1.98 & $0.49 \pm 0.16$ \\
BB-pol & 1.09 & $0.61 \pm 0.07$ \\
edep & 0.81 & $0.62 \pm 0.06$ \\
pol & 0.48 & $0.53 \pm 0.01$ \\
\hline
\end{tabular}

* Abbreviations: $\mathrm{BB}=$ bead-blasted $\mathrm{Ni}$; edep = electrodeposited $\mathrm{Ni}$; pol = polished $\mathrm{Ni}$.

${ }^{\dagger}$ Cathodic peaks were integrated to determine the quantities of charge passed $\left(Q_{\text {cat }}\right)$ from the last scan in the $\mathrm{CV}$ plots acquired during the steady-state aging treatment.

* Average quantities of charge passed were determined from the last CV scan for each replicate electrode. The error represents one standard deviation from the calculated mean values. 

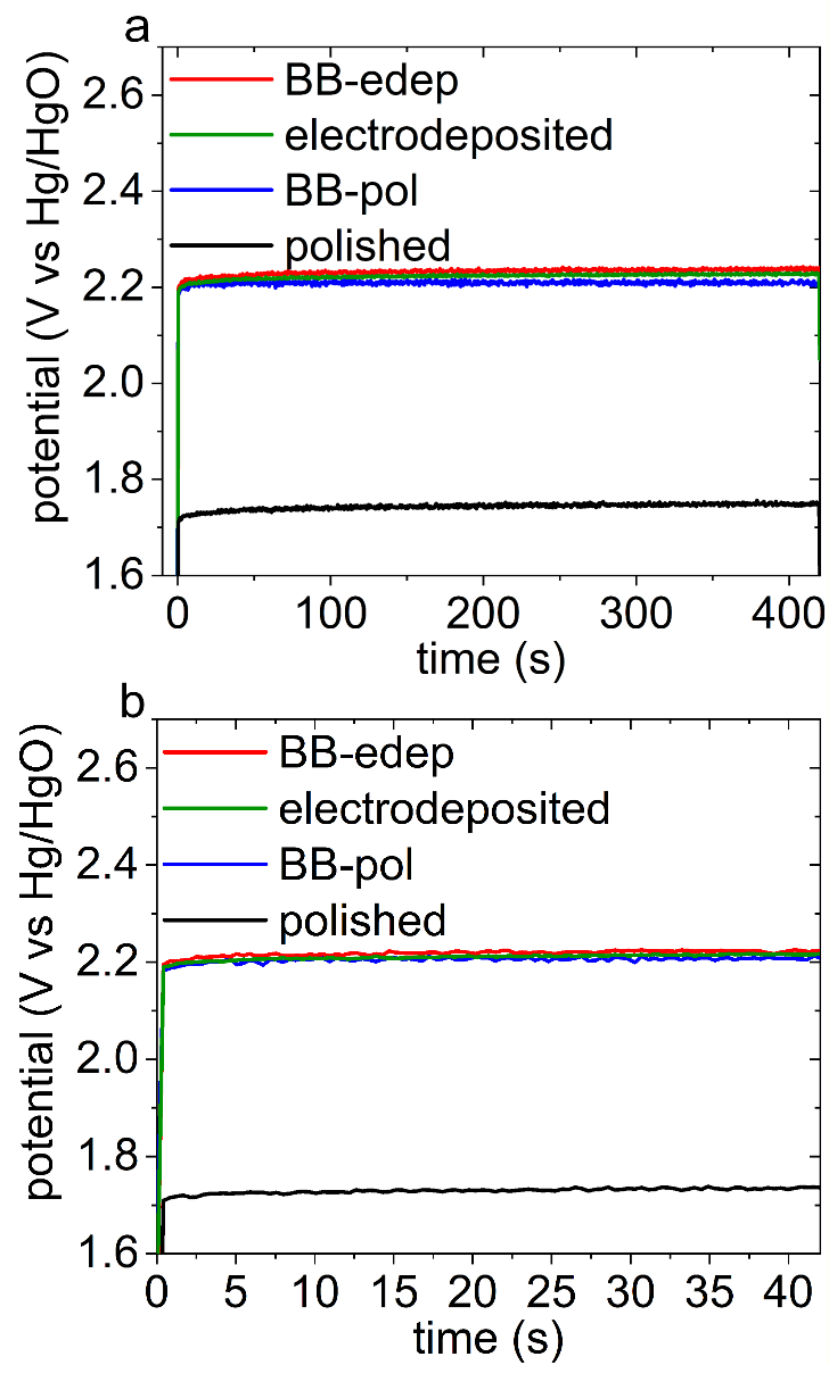

Figure S17. Chronopotentiometry (CP) for Ni electrodes treated with the limited aging process. The data was acquired at $500 \mathrm{~mA} / \mathrm{cm}^{2}$ in purified $1 \mathrm{M} \mathrm{KOH}$ at $25^{\circ} \mathrm{C}$. Each transient is plotted as an average of three replicates. The plots in (a) were acquired at $3000 \mathrm{rpm}$. The plots in (b) show the results over a shorter time-scale, corresponding to the data plotted in (a). 

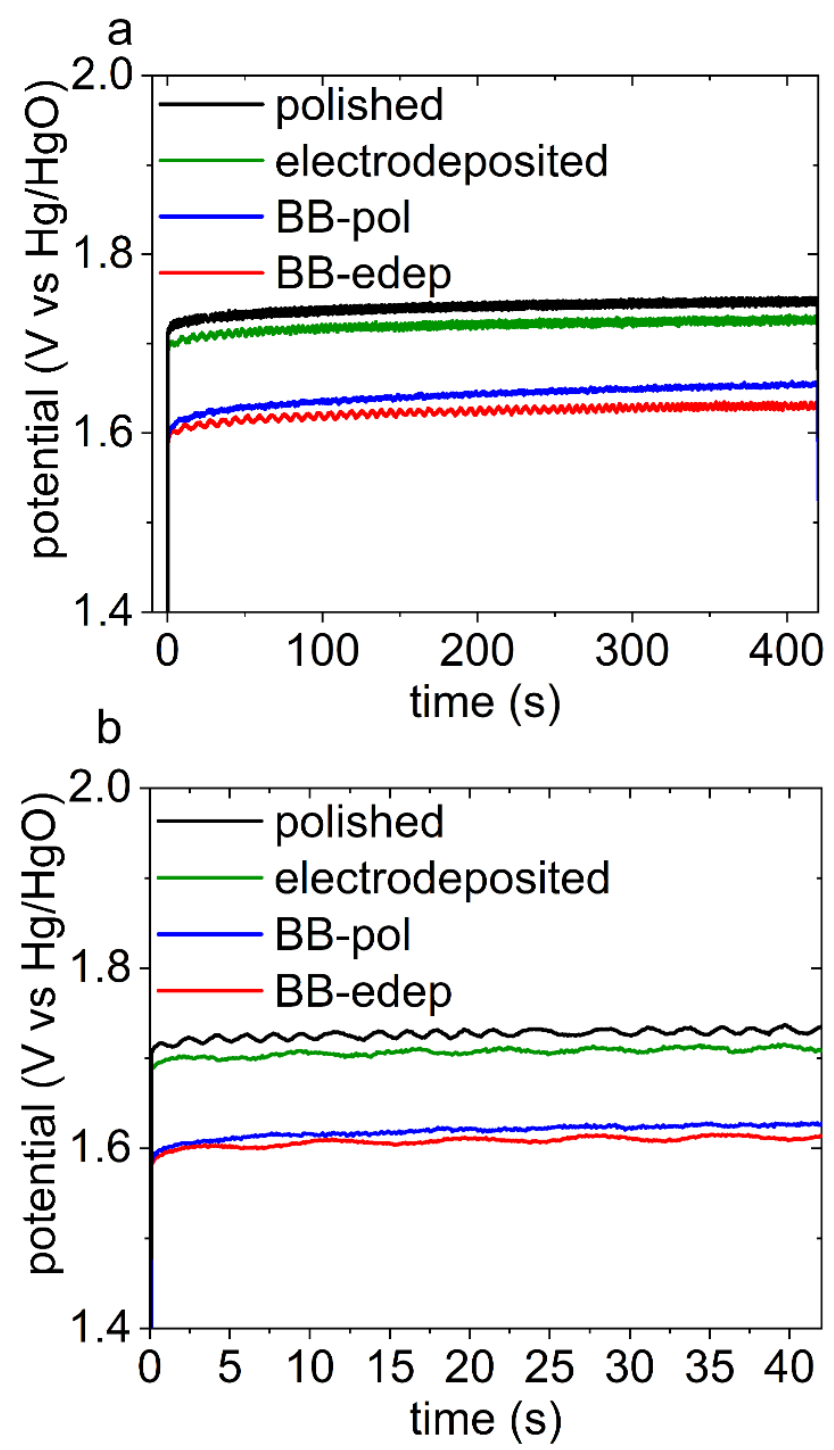

Figure S18. Representative CP data acquired at $500 \mathrm{~mA} / \mathrm{cm}^{2}$ after completing a steady-state aging process for each type of $\mathrm{Ni}$ electrode in purified $1 \mathrm{M} \mathrm{KOH}$ at $25^{\circ} \mathrm{C}$. The plot in (a) was acquired at $3000 \mathrm{rpm}$. The plot in (b) shows the CP data over a shorter time-scale than in (a). 

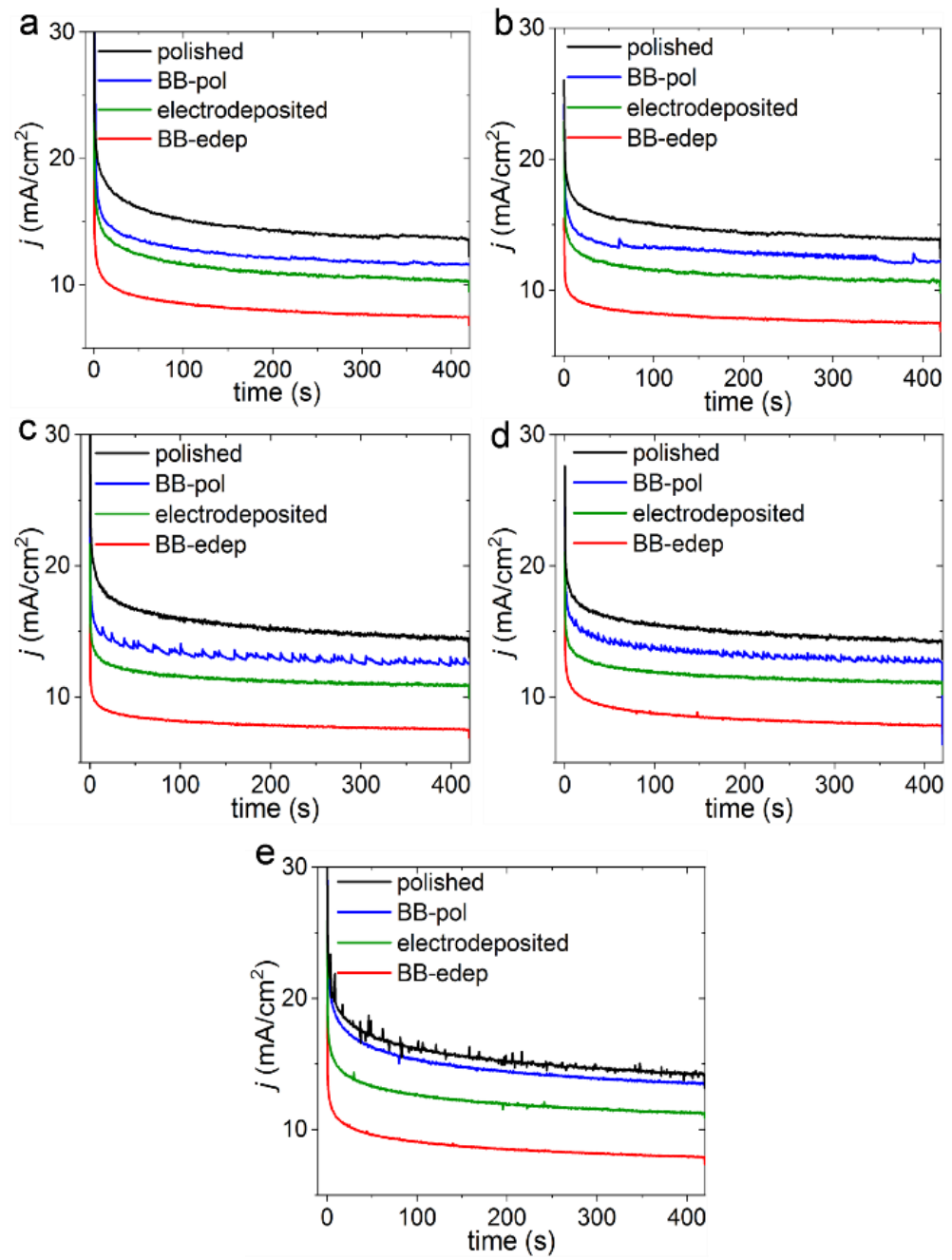

Figure S19. Chronoamperometry (CA) measurements obtained at $1.0 \mathrm{~V}(\mathrm{vs} \mathrm{Hg} / \mathrm{HgO}$ ) for $\mathrm{Ni}$ electrodes treated with the limited aging process in purified $1 \mathrm{M} \mathrm{KOH}$ at $25^{\circ} \mathrm{C}$. Each set of plots were acquired at different rotational speeds: (a) $500 \mathrm{rpm}$; (b) $1000 \mathrm{rpm}$; (c) $2000 \mathrm{rpm}$; (d) 3000 rpm; and (e) $7000 \mathrm{rpm}$. Each transient is an average of three individual replicates, and currents were normalized to the theoretical $\mathrm{SA}_{\mathrm{f}}$. 

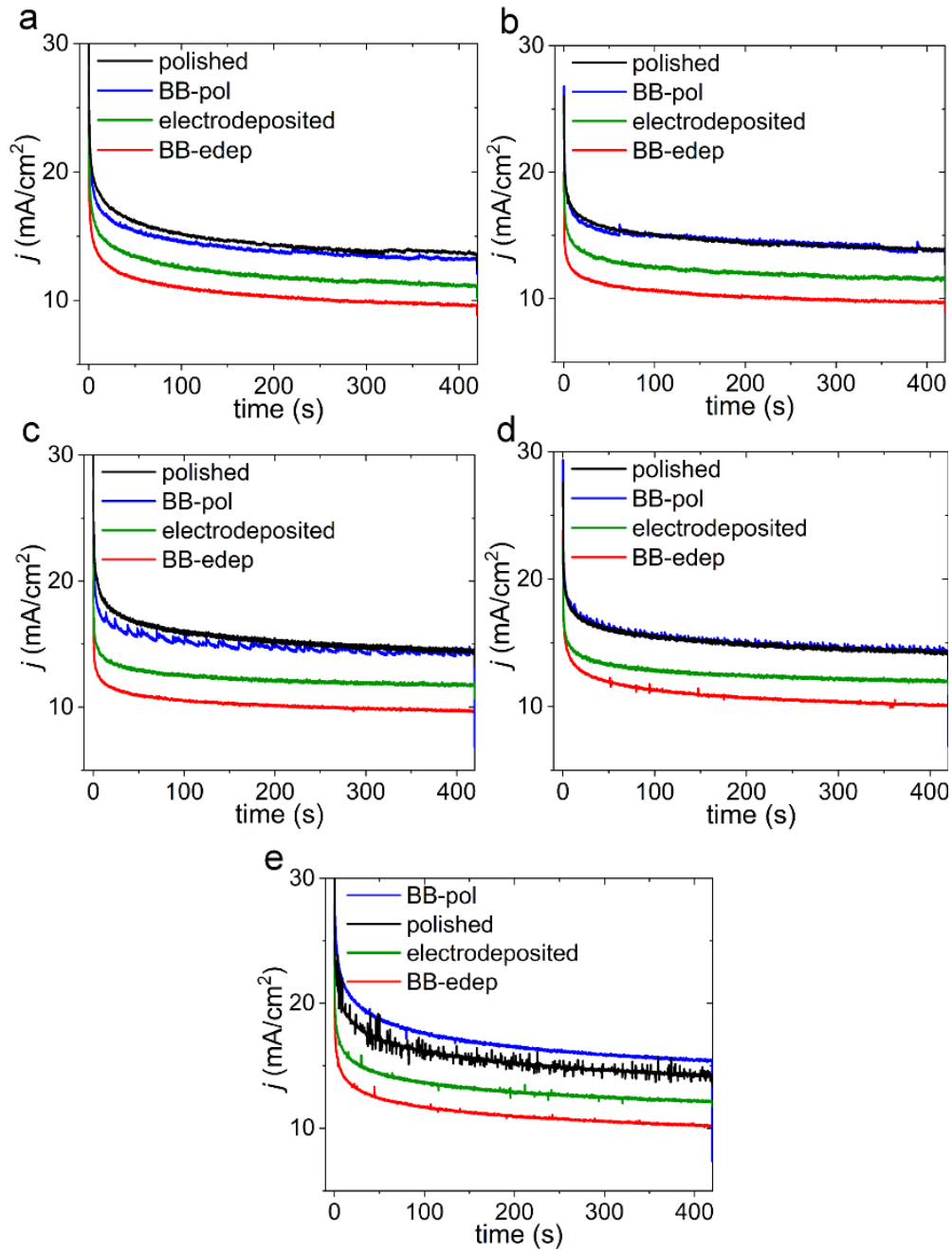

Figure S20. Chronoamperometry (CA) measurements obtained at $1.0 \mathrm{~V}(\mathrm{vs} \mathrm{Hg} / \mathrm{HgO})$ for $\mathrm{Ni}$ electrodes treated with the limited aging process in purified $1 \mathrm{M} \mathrm{KOH}$ at $25^{\circ} \mathrm{C}$. Each set of plots were acquired at different rotational speeds: (a) $500 \mathrm{rpm}$; (b) $1000 \mathrm{rpm}$; (c) $2000 \mathrm{rpm}$; (d) 3000 rpm; and (e) $7000 \mathrm{rpm}$. Each transient is an average of three individual replicates, and currents were normalized to the geometric area of the electrodes. 

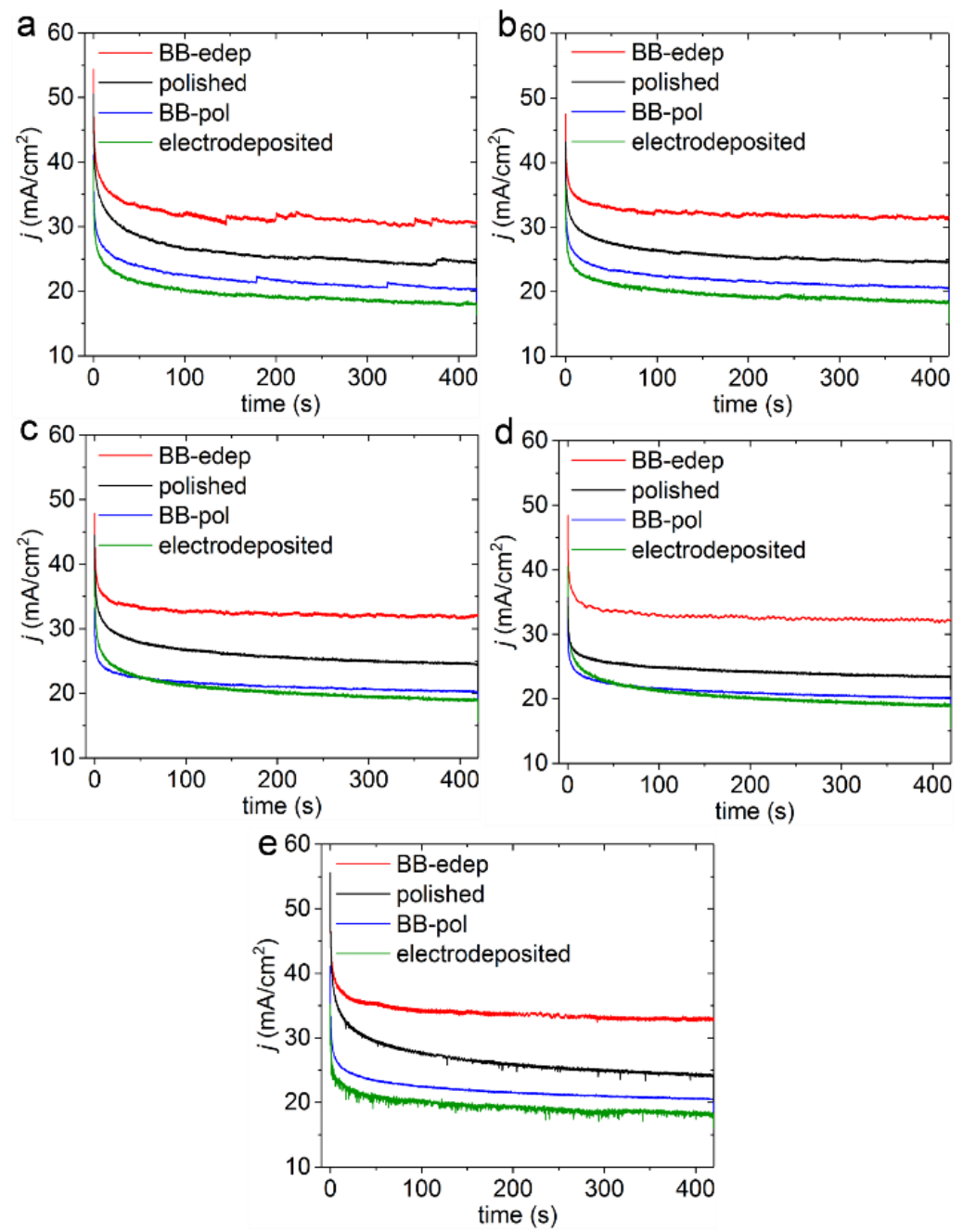

Figure S21. Representative CA data acquired at $1.0 \mathrm{~V}$ versus $\mathrm{Hg} / \mathrm{HgO}$ for each type of textured $\mathrm{Ni}$ electrode after a steady-state aging process in purified $1 \mathrm{M} \mathrm{KOH}$ at $25{ }^{\circ} \mathrm{C}$. Each plot was acquired at different rotational speeds: (a) 500 rpm; (b) $1000 \mathrm{rpm}$; (c) 2000; (d) 3000; and (e) 7000 $\mathrm{rpm}$. The currents were normalized to the theoretical $\mathrm{SA}_{\mathrm{f}}$ for each sample. 

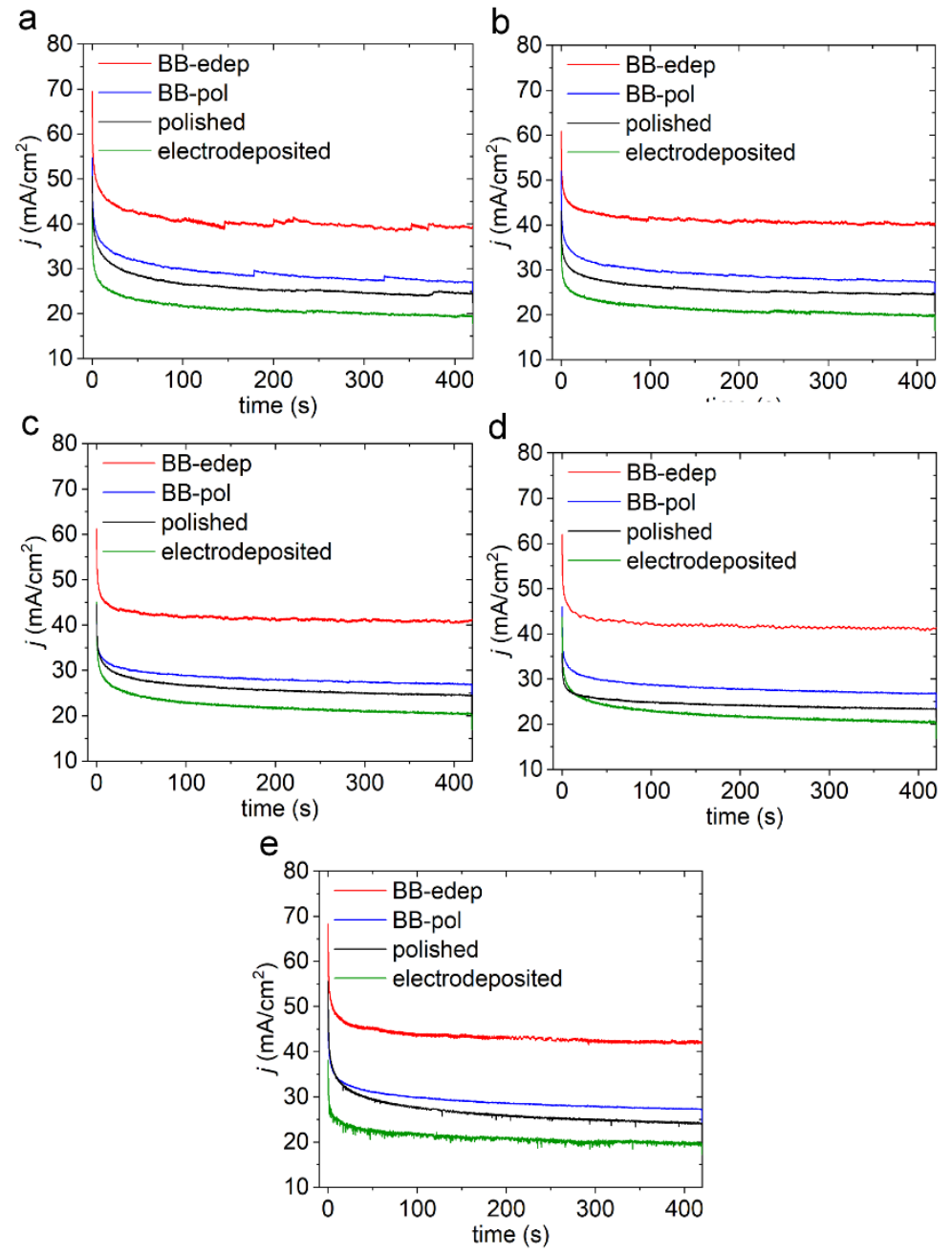

Figure S22. Representative CA data acquired at $1.0 \mathrm{~V}$ versus $\mathrm{Hg} / \mathrm{HgO}$ for each type of textured $\mathrm{Ni}$ electrode after a steady-state aging process in purified $1 \mathrm{M} \mathrm{KOH}$ at $25{ }^{\circ} \mathrm{C}$. Each plot was acquired at different rotational speeds: (a) $500 \mathrm{rpm}$; (b) $1000 \mathrm{rpm}$; (c) 2000; (d) 3000; and (e) 7000 rpm. The currents were normalized to the geometric area of the electrodes. 

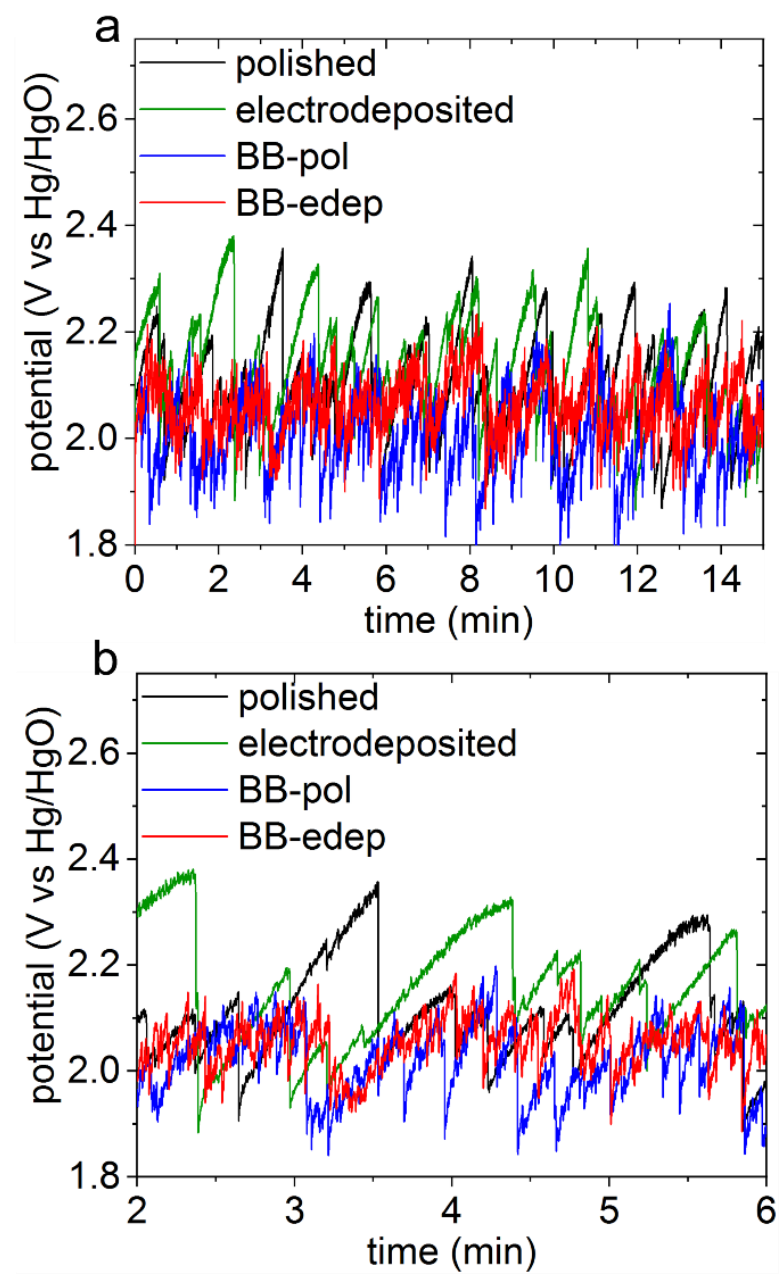

Figure S23. (a) Representative CP data obtained at $500 \mathrm{~mA} / \mathrm{cm}^{2}$ for electrodes in a face-up configuration without electrode rotation in $1 \mathrm{M} \mathrm{KOH}$ after completing the steady-state aging process. The data in (b) is shown for a shorter time-scale corresponding to the trends observed in (a). 


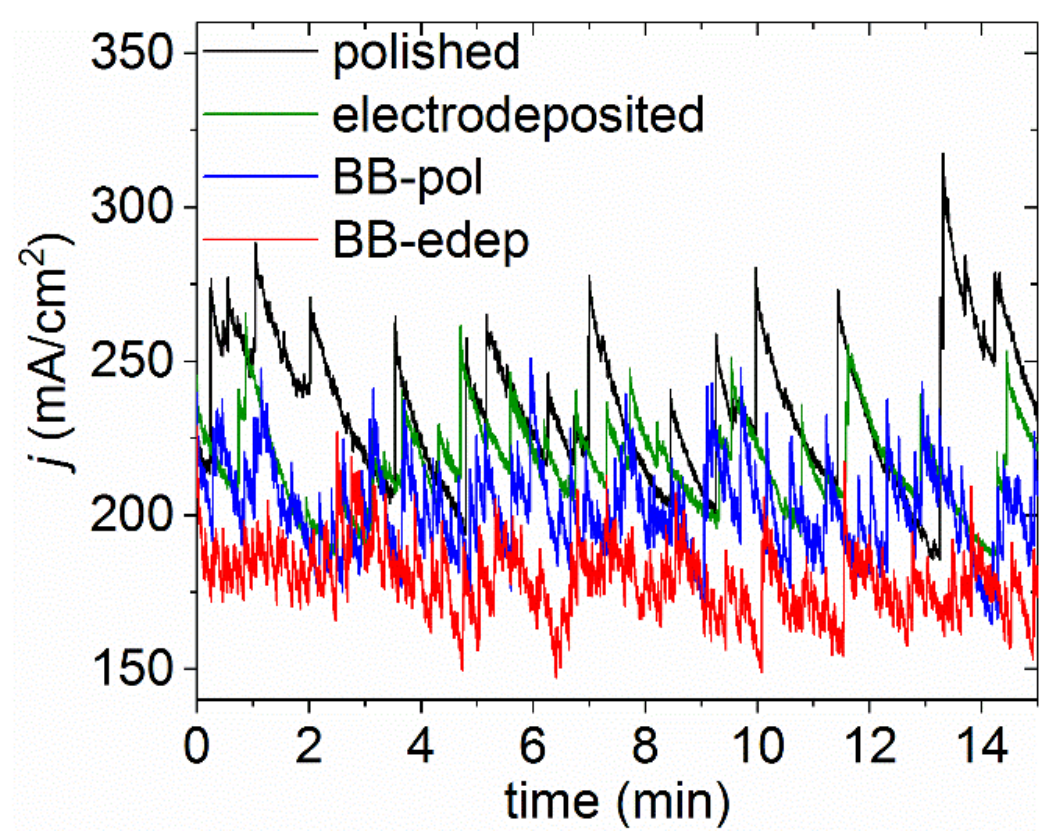

Figure S24. Representative CA transients acquired at $1.5 \mathrm{~V}$ (versus $\mathrm{Hg} / \mathrm{HgO}$ ) for $\mathrm{Ni}$ electrodes treated with the steady-state aging process. The electrodes were held in a face-up configuration without rotation. The measured currents were normalized against the theoretical $\mathrm{SA}_{\mathrm{f}}$ for each electrode. 


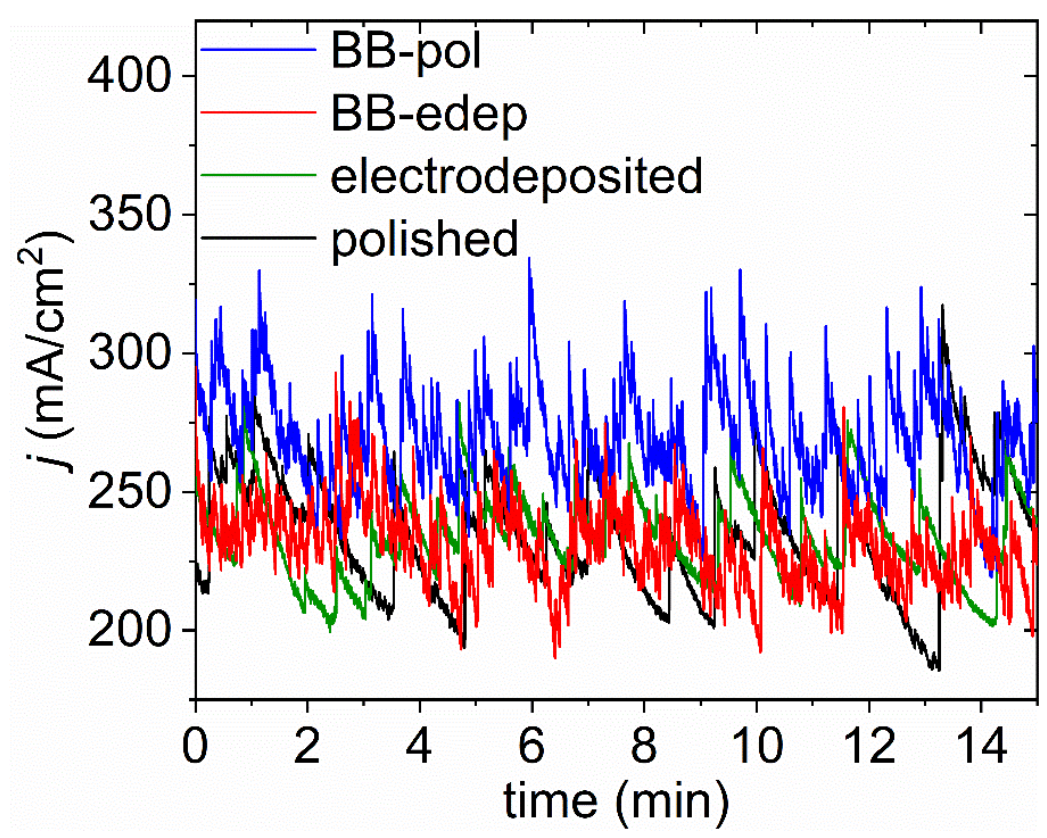

Figure S25. Representative CA transients acquired at $1.5 \mathrm{~V}$ (versus $\mathrm{Hg} / \mathrm{HgO}$ ) for $\mathrm{Ni}$ electrodes treated with the steady-state aging process. The electrodes were held in a face-up configuration without rotation. The measured currents were normalized against the geometric area of the electrodes. 

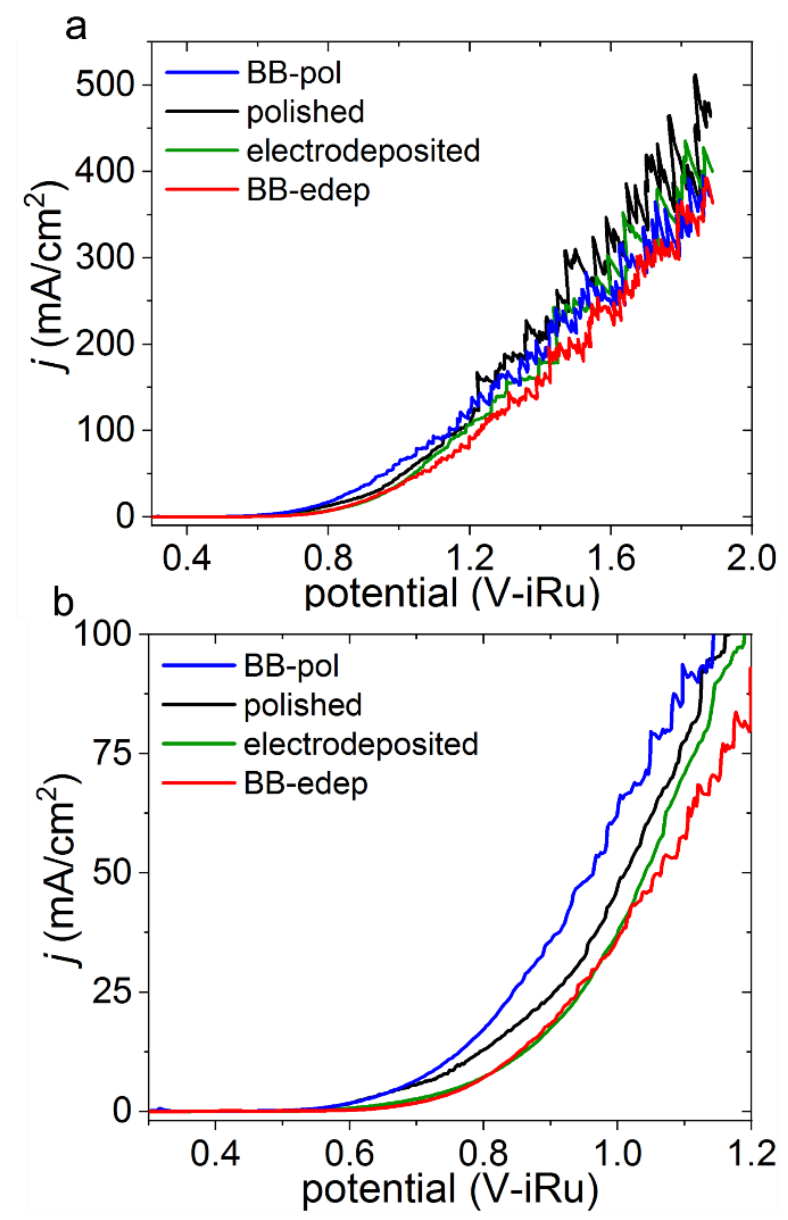

Figure S26. The LSV plots obtained for each of the Ni electrodes while oriented in a face-up configuration without any rotation. The data were recorded at a scan rate of $1 \mathrm{mV} / \mathrm{s}$ in high purity $1 \mathrm{M} \mathrm{KOH}$ at $25{ }^{\circ} \mathrm{C}$ after performing the steady-state aging process for each type of electrode. The potential was scanned in (a) from 0 to $2.0 \mathrm{~V}$ ( $\mathrm{vs} \mathrm{Hg} / \mathrm{HgO}$ ). The plot in (b) shows the current density as a function of scanning the potential from 0 to $1.2 \mathrm{~V}(\mathrm{vs} \mathrm{Hg} / \mathrm{HgO})$. The observed currents were normalized to the theoretical $\mathrm{SA}_{\mathrm{f}}$ for each electrode. 

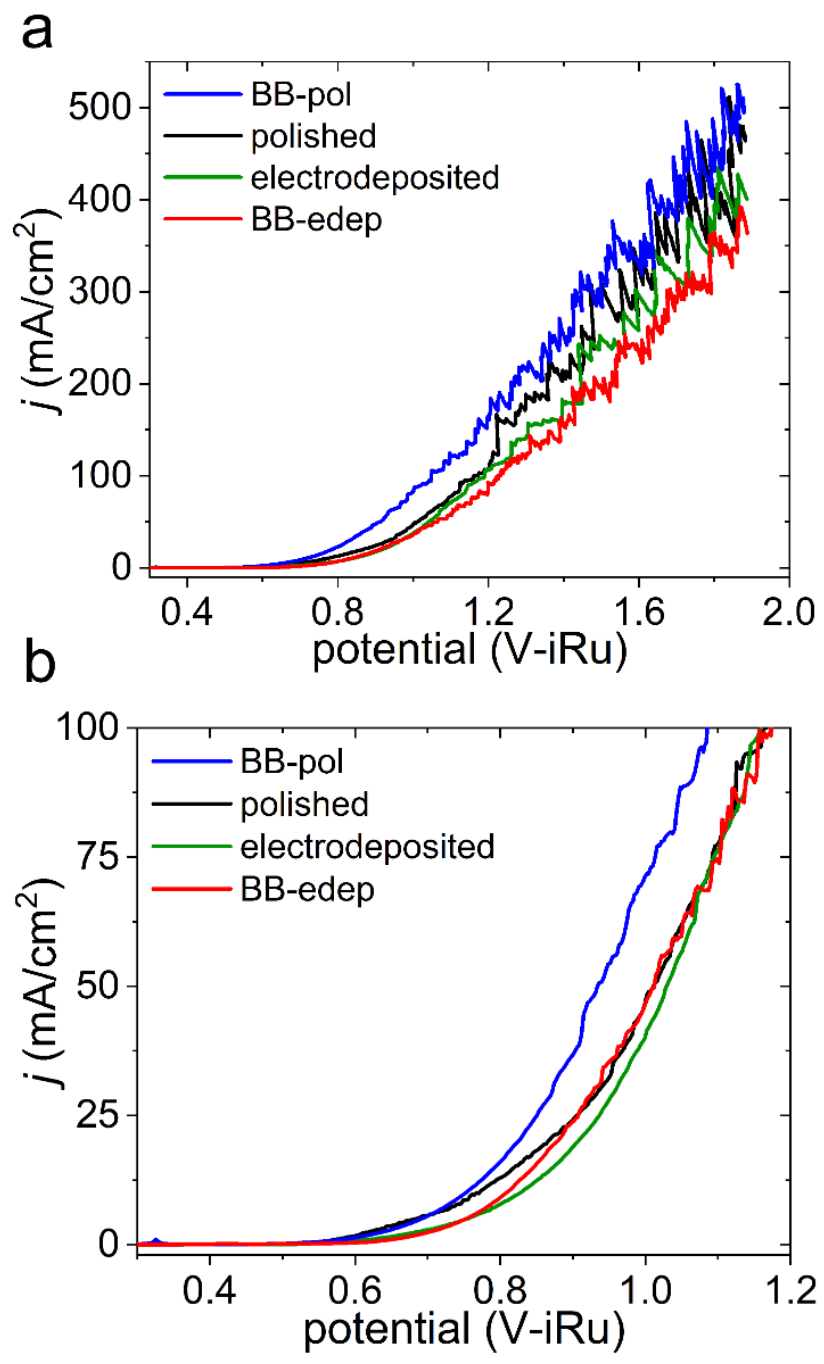

Figure S27. The LSV plots obtained for each of the Ni electrodes while oriented in a face-up configuration without any rotation. The data were recorded at a scan rate of $1 \mathrm{mV} / \mathrm{s}$ in high purity $1 \mathrm{M} \mathrm{KOH}$ at $25{ }^{\circ} \mathrm{C}$ after performing the steady-state aging process for each type of electrode. The potential was scanned in (a) from 0 to $2.0 \mathrm{~V}$ (vs $\mathrm{Hg} / \mathrm{HgO}$ ). The plot in (b) shows the current density as a function of scanning the potential from 0 to $1.2 \mathrm{~V}(\mathrm{vs} \mathrm{Hg} / \mathrm{HgO})$. The observed currents were normalized to the geometric area of the electrodes. 


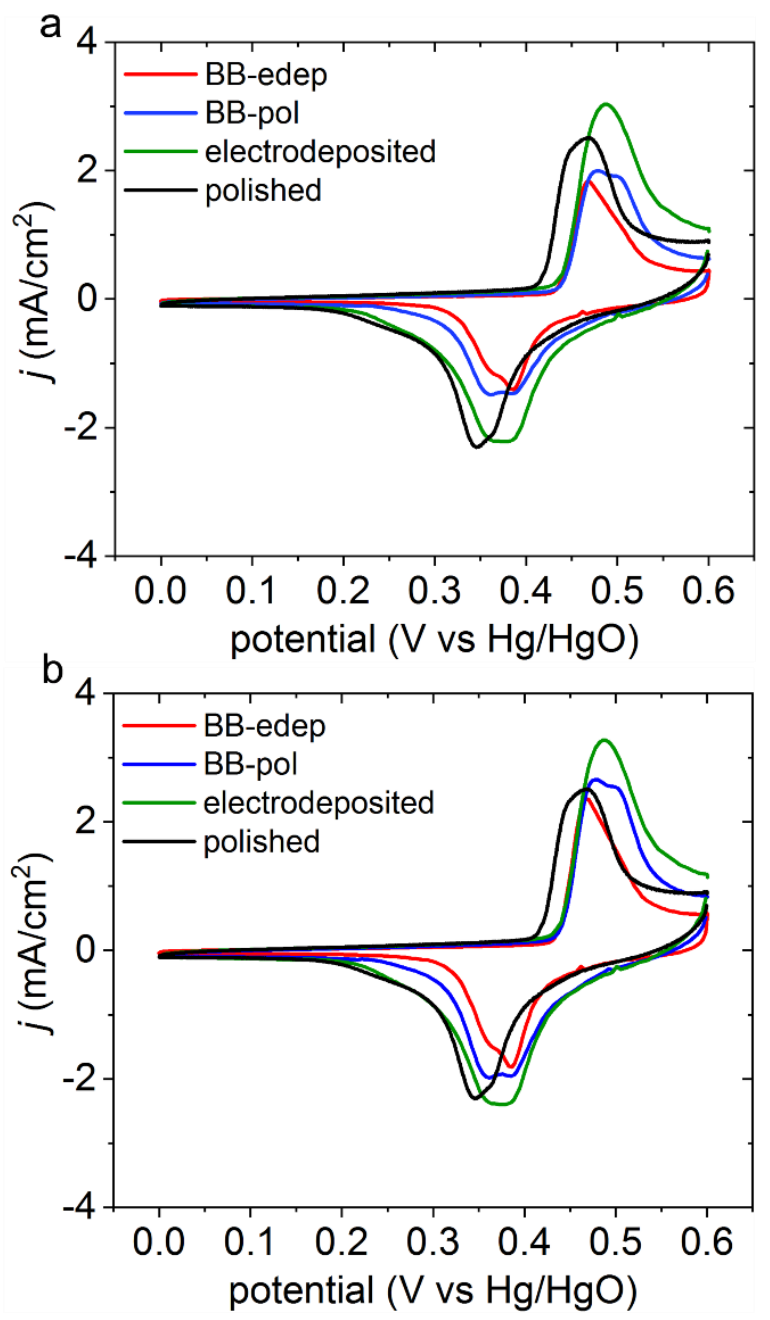

Figure S28. The $100^{\text {th }} \mathrm{CV}$ scan resulting from the limited aging process for each type of electrode. The data were normalized against (a) the $\mathrm{SA}_{\mathrm{f}}$, and (b) the geometric surface area. All $\mathrm{CV}$ profiles were acquired at $100 \mathrm{mV} / \mathrm{s}$ in purified $1 \mathrm{M} \mathrm{KOH}$ at $25{ }^{\circ} \mathrm{C}$ with an electrode rotational speed of 5500 rpm. 


\section{References}

(1) Gerson, A.; Biesinger, M. C.; Smart, R. S. C.; Payne, B. P.; Lau, L. W. M. X-Ray Photoelectron Spectroscopic Chemical State Quantification of Mixed Nickel Metal, Oxide and Hydroxide Systems. Surf. Interface Anal. 2009, 41, 324-332.

(2) Grosvenor, A. P.; Biesinger, M. C.; Smart, R. S. C.; McIntyre, N. S. New Interpretations of XPS Spectra of Nickel Metal and Oxides. Surf. Sci. 2006, 600, 1771-1779. 